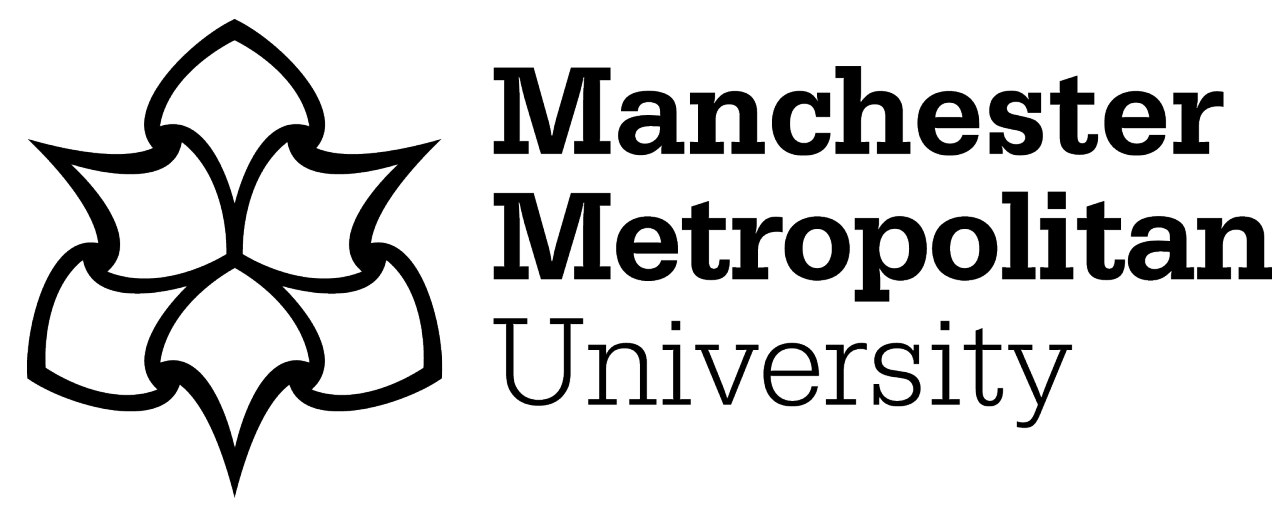

Mole, CD, Kountouriotis, G ORCID logoORCID: https://orcid.org/0000-00026346-9258, Billington, J and Wilkie, RMG (2016) Optic flow speed modulates guidance level control: New insights into two-level steering. Journal of Experimental Psychology: Human Perception and Performance, 42 (11). pp. 1818-1838. ISSN 0096-1523

Downloaded from: https://e-space.mmu.ac.uk/626741/

Version: Accepted Version

Publisher: American Psychological Association

DOI: https://doi.org/10.1037/xhp0000256

Please cite the published version 
Running Head: Optic Flow modulates Guidance Steering Control

\section{Optic flow speed modulates guidance level control: new insights into}

\section{two-level steering.}

Mole, Callum David ${ }^{1}$, Kountouriotis, Georgios ${ }^{2}$, Billington, Jac ${ }^{1} \&$ Wilkie, Richard McGilchrist $^{1^{*}}$

*Corresponding author

${ }^{1}$ School of Psychology.

${ }^{2}$ Institute of Transport studies 
Running Head: Optic Flow modulates Guidance Steering Control

\section{Abstract}

Responding to changes in the road ahead is essential for successful driving. Steering control can be modelled using two complementary mechanisms: guidance control (to anticipate future steering requirements) and compensatory control (to stabilise position-in-lane). Drivers seem to rapidly sample the visual information needed for steering using active gaze patterns, but the way in which this perceptual information is combined remains unclear. Influential models of steering capture many steering behaviours using just 'far' and 'near' road regions to inform guidance and compensatory control respectively (Salvucci \& Gray, 2004). However, optic flow can influence steering even when road-edges are visible (Kountouriotis et al., 2013). Two experiments assessed whether flow selectively interacted with compensatory and/or guidance levels of steering control, under either unconstrained gaze or constrained gaze conditions. Optic flow speed was manipulated independent of the veridical road-edges so that use of flow would lead to predictable understeering or oversteering. Steering was found to systematically vary according to flow speed, but crucially the Flow-Induced Steering Bias (FISB) magnitude depended on which road-edge components were visible. The presence of a guidance signal increased the influence of flow, with the largest FISB in 'Far' and 'Complete' road conditions, whereas the smallest FISB was observed when only 'Near' road-edges were visible. Gaze behaviours influenced steering to some degree, but did not fully explain the interaction between flow and road-edges. Overall the experiments demonstrate that optic flow can act indirectly upon steering control by modulating the guidance signal provided by a demarcated path. 
Running Head: Optic Flow modulates Guidance Steering Control

\section{Introduction}

Humans are skilled at controlling self-motion, and they perform locomotor tasks so effortlessly that it is easy to underestimate the complexity of the problem posed to the human perceptual-motor system. Successful locomotion relies on identifying a collision-free course to the environmental goal (using information such as optic flow; Gibson, 1958), exerting mechanical forces to propel the organism (or vehicle) down the chosen path, and using sensory feedback to dynamically respond to errors. Moreover, it is routinely the case that locomotor trajectories are constrained by lane boundaries: be it forest trail, pavement, country lane, or motorway. This paper examines how humans use optic flow and road-edge information to steer along such demarcated paths.

Consider driving down a single track road. This task can be achieved by simple feedback control. The first step requires the driver to merely move themselves forward (by pressing the accelerator/gas pedal). At the next moment, the driver can perceive their current position using splay rate (e.g. Beall \& Loomis, 1996) or egocentric direction (Salvucci \& Gray, 2004) relative to the near road-edges and compare that to their ideal position (e.g. centre of the road). The final step requires the driver to produce a motor command that reduces the error signal to zero. These steps can be repeated in what could be a continuous loop, enabling the driver to correct for internal disturbances (e.g. signalling noise; Faisal, Selen, \& Wolpert, 2008) or external disturbances (e.g. crosswind) in order to reduce trajectory error to acceptable limits.

This feedback control strategy requires the driver to continuously monitor the road. In real world scenarios, however, it is unlikely that such dedicated monitoring is possible, with the driver's attention distributed across the scene (e.g. to monitor road signs, passengers, 
Running Head: Optic Flow modulates Guidance Steering Control

pedestrians and other cars) so that information is only sampled intermittently from the road (Godthelp, 1986; Senders et al., 1967). There are also inherent delays that limit the usefulness of a feedback control strategy (e.g. system delays due to second-order control ${ }^{1}$ ). At slow driving speeds errors develop only gradually, therefore feedback mechanisms can be used at a low gain to smoothly correct for errors. As driving speeds increase, however, there comes a point at which errors develop so quickly that gradual correction is not possible. The driver now has to make large error corrections to compensate for the rapid error growth at each moment. Under these conditions a feedback control strategy results in oscillatory trajectories and jerky steering, so compensatory feedback based on lane position is no longer sufficient to provide adequate control (Land \& Horwood, 1995).

Fortunately, there are alternatives to pure compensatory control. Humans have a mobile gaze system that allows distant information to be sampled in order to anticipate future steering requirements (feedforward control). In principle such prospective information could be sufficient to steer (without need for immediate error feedback); however, these prospective signals tend to be less indicative of the immediate positional error. Optimal motor control would, therefore, make use of both feedforward and feedback control systems, with the feedforward component reducing delays and error thus allowing feedback to operate at a lower gain (cf. Desmurget \& Grafton, 2000). Drawing on these principles, Donges (1978) proposed that steering consisted of both an anticipatory process and a compensatory (error

\footnotetext{
${ }^{1}$ Second order control, or acceleration control, means there are two integrations between the control input and device output. Change in steering wheel angle corresponds to a change in lateral acceleration (heading velocity), which integrates to specify lateral velocity (heading), which integrates to specify position. Therefore, there is a delay inherent in the control system between input (change in steering wheel angle) and device output (Jagacinski \& Flach, 2003).
} 
Running Head: Optic Flow modulates Guidance Steering Control

correction) process. The anticipatory mechanism specifies future steering requirements, and the compensatory mechanism monitors positional error (see Figure 1D).

Often the terms feedforward, open-loop, prospective, anticipatory and guidance are used interchangeably (Donges, 1978; Land, 1998). As a result, feedforward (open-loop) control is often conflated with the use of information specified by a far point for feedback (closed-loop) control. This is a non-trivial confusion. Although some two-level models treat guidance control as a parallel, open-loop, process (e.g. Markkula, Benderius, \& Wahde, 2014; for reviews see Plöchl \& Edelmann, 2007; Steen, Damveld, Happee, van Paassen, \& Mulder, 2011), evidence suggests that the internal models for open-loop steering control are often poor (Cloete \& Wallis, 2009; Macuga, Beall, Kelly, Smith, \& Loomis, 2007). In contrast, there is extensive literature examining how perceptual variables are 'picked up' from the scene and used to adjust steering towards some goal under the tacit framework of feedback control (e.g. Salvucci \& Gray, 2004; Wilkie, Wann, \& Allison, 2008; see also Mars, Saleh, Chevrel, Claveau, \& Lafay, 2011; Saleh, Chevrel, Mars, Lafay, \& Claveau, 2011; Sentouh, Chevrel, Mars, \& Claveau, 2009). To avoid further confusion, this manuscript uses the terms originally proposed by Donges (1978) of guidance control to describe steering using information from a far point, and compensatory control to describe steering using information from a near point.

One of the most commonly cited two-level steering models in the vision science literature is Salvucci \& Gray's two point visual control model (2004). Salvucci and Gray (2004) reformulated Donges's (1978) two-level approach into a 'Proportional-Integral' feedback controller using egocentric direction from a far point and a near point. This model acts to keep the egocentric direction of the near and far points stable (the proportional part), whilst simultaneously reducing error of the near point relative to some ideal reference (e.g. centre 
Running Head: Optic Flow modulates Guidance Steering Control

of the road; the integral part). Salvucci \& Gray (2004) proposed that by simply changing the location of the near or far point, or changing the weights attributed to each component, such a model can account for various steering behaviours, such as curve negotiation, corrective steering, lane changing, and also individual differences in steering strategy. Consistent with the two-level predictions, the far point acts to smoothly guide steering but it does not necessarily maintain lateral position in the centre of the road. And while the near point acts to keep the vehicle in the lane centre, without the far region to provide anticipatory information about changing steering requirements it would be expected to produce rapid oscillatory "bang-bang" steering (Salvucci \& Gray, 2004).

Whilst such a two-level dichotomy is appealing, there is only weak empirical evidence to support it. One highly influential study (Land \& Horwood, 1995) asked participants to steer a central trajectory whilst road-edge information was restricted using either one or two $1^{\circ}$ viewing 'windows'. When one viewing window displayed the far region and another one displayed the near region, the paper reported that steering performance was equivalent to full road conditions. It was suggested, therefore, that steering can be accurate as long as both a guidance and compensatory signal are available. When only one viewing window was available, an 'optimum' region was identified midway between the far and near regions, suggesting that having either a compensatory or guidance signal alone is not sufficient for accurate steering. Consistent with the Donges' (1978) predictions of two-level control, when the near road was available steering became jerky, and when only far road was available steering became smoother but less accurate.

Unfortunately there has been a failure to replicate some of Land \& Horwood's (1995) key conclusions. Using similar road-edge manipulations to Land \& Horwood (1995), Chatziastros, 
Running Head: Optic Flow modulates Guidance Steering Control

Wallis, \& Bülthoff (1999) and Cloete \& Wallis (2011) fail to find evidence for a 'half-way optimum'. Additionally, Chatziastros et al. (1999) found that the addition of a fixed far region did not improve steering accuracy nor did it increase stability. Cloete \& Wallis (2011) failed to replicate a decrease in steering accuracy when the near road was removed, nor did they observe equivalent performance to full road conditions when one viewing window displayed the far region and the other one displayed the near region. Taken together, these studies question the core results of Land \& Horwood (1995). Cloete \& Wallis (2011) suggested that the low display refresh rate in Land \& Horwood's study $(7 \mathrm{~Hz})$ may have exacerbated some of the observed steering effects. Comparing both lower and higher refresh rates $(7.2 \mathrm{~Hz}$ and $72 \mathrm{~Hz}$ ), Cloete \& Wallis (2011) found that steering behaviour was less accurate (measured by standard deviation of the deviation from the road centre) and jerkier at the lower refresh rate, and that this effect was especially pronounced when only a near road segment was viewed. They did, however, replicate the finding that steering behaviour becomes increasingly jerky as guidance level information is removed (this interaction is reduced in $72 \mathrm{~Hz}$ displays compared with the $7.2 \mathrm{~Hz}$ refresh rate, but still consistent across participants; Cloete \& Wallis, 2011).

Although both Chatziastros et al. (1999) and Cloete \& Wallis (2011) question Land \& Horwood's (1995) findings of a mid-road optimum and that adding guidance level information improves steering performance, these studies do still support the two-level model prediction that compensatory control (using information from near regions) leads to accurate steering at the expense of jerkier behaviour (at least at low speeds; cf. Frissen \& Mars, 2014). More recent support for the two-level model comes from Frissen \& Mars (2014), who applied an opacity mask $(20 \%, 40 \%, 60 \%, 80 \%$, or $100 \%$ opaque) to either the top half (which contained 
Running Head: Optic Flow modulates Guidance Steering Control

some far road-edge information, as well as sky) or bottom half (which contained only near road-edge information) of the display, thus degrading guidance or compensatory level information respectively. Removing compensatory information led to an increase in steering variability (measured by standard deviation of lane position) although mean lane position and steering smoothness were no different to baseline performance. This agrees with the twolevel proposal that steering with guidance level information tends to be smooth but imprecise (Land, 1998). Removing guidance information, however, led to jerkier steering which was also more variable and less accurate (i.e. there were large errors in mean lane position) than baseline. This is consistent with the notion that compensatory control leads to accurate steering at the expense of jerky behaviour (Donges, 1978). Although the two-level model may lack definitive empirical support, a two-level approach to explaining steering control is now commonplace amongst computational models of steering behaviour (Markkula et al., 2014; Plöchl \& Edelmann, 2007), and appears to be a useful way of describing the nature of the control task of driving when the path to be followed is demarcated by visual features.

An aspect of human behaviour that is often overlooked when considering steering is the way in which gaze patterns can inform and/or interact with the information available from the visual scene. Two-level models usually posit that the driver looks at a far point to retrieve anticipatory information, whilst position-in-lane is monitored through peripheral vision (Kountouriotis, Floyd, Gardner, Merat, \& Wilkie, 2012; Salvucci \& Gray, 2004), but they do not always make specific predictions about where a driver should look to obtain the anticipatory information. Candidate locations for where gaze falls during curve driving can be broadly grouped into tangent point (TP) fixation or future path (FP) fixation (cf. Mars \& Navarro, 2012; for a critical review, see Lappi, 2014). TP fixation has been reported in a number of on-road 
Running Head: Optic Flow modulates Guidance Steering Control

studies (Chattington, Wilson, Ashford, \& Marple-Horvat, 2007; Kandil, Rotter, \& Lappe, 2009, 2010; Underwood, Chapman, Crundall, Cooper, \& Wallen, 1999; Land \& Lee, 1994). However, these studies have generally evaluated gaze data using an area of interest (Aol) approach, and Lappi (2014) has raised a number of shortcomings of using Aol methodology to disentangle FP vs. TP fixations. Using more sophisticated methods of localising gaze during naturalistic driving (e.g. using the pattern of opto-kinetic nystagmus to identify the regions of local flow in which gaze falls), Lappi and colleagues have provided fresh evidence that the anticipatory information required for high-speed steering tends to come from a point on the FP next to, or beyond, the TP, rather than from the TP itself (Itkonen, Pekkanen, \& Lappi, 2015; Lappi, Pekkanen, \& Itkonen, 2013; Lappi \& Pekkanen, 2013; see also Mars \& Navarro, 2012).

This proposal fits well within the Active Gaze Model of steering (Wilkie et al., 2008) which predicts that one should look where one is going during curved driving (i.e. toward a far point on the desired FP). The Active Gaze Model is based on the principles of using the fixation as a point-attractor that supplies multiple sources of information (Wilkie \& Wann, 2002) to maintain robust steering control (see also Fajen \& Warren, 2003, for a similar approach). The model uses perceptual inputs from the retinal flow field as well as the rate of change of extraretinal direction (e.g. gaze) and retinal direction (e.g. the bonnet/hood of the car). While it appears that the Active Gaze Model captures gaze behaviours during steering curved trajectories fairly well (Wilkie et al., 2008), it should be noted that the Active Gaze Model was conceived to explain the most general of steering behaviours where trajectories can be fairly unconstrained (Wilkie \& Wann, 2002, 2003a) rather than specifically for the special case of driving along a road ${ }^{2}$. Conversely, models of steering constrained trajectories have tended to

\footnotetext{
${ }^{2}$ It is currently unclear precisely how the Active Gaze Model maps onto two-level control. One may intuit that since perceptual inputs are obtained by looking towards a far point the Active Gaze Model would support
} 
Running Head: Optic Flow modulates Guidance Steering Control

ignore the utility of retinal flow information and instead focus on road-edges as the sole source of relevant information (Donges, 1978; Salvucci \& Gray, 2004). This is perhaps understandable since the lane boundaries actually define the steering task requirements, however, there is a large body of literature highlighting the importance of optical/retinal flow information for the successful control of locomotion (e.g. Cheng \& Li, 2011; Kountouriotis et al., 2013; Li \& Chen, 2010; Saunders \& Ma, 2011; Warren, Kay, Zosh, Duchon, \& Sahuc, 2001; Wilkie \& Wann, 2002), and there are good theoretical reasons to suppose that flow information will remain useful when looking at either the TP (Authié \& Mestre, 2012; Raviv \& Herman, 1991) or the FP (Kim \& Turvey, 1999; Wann \& Swapp, 2000). Whilst Land \& Horwood (1995) did not appear to specifically set out to exclude flow, their display did not contain textured surfaces which limited flow information. Salvucci \& Gray's (2004) model is specific to near and far road-edges, with little specific role for flow since perceiving the visual direction of the road-edges is not usually reliant upon flow information. Similarly, Beall \& Loomis (1996) argue that if splay angle information from road markers is available, retinal flow does little to contribute to performance. Furthermore, road-edges seem to strongly influence where the eyes are directed during curve driving (Kountouriotis et al., 2012; Land \& Lee, 1994) which suggests that the road-edge information is often highly weighted as an input to steering control.

The implicit assumption, then, is that when steering along a road there is little use for retinal flow. However, such a statement does not fit well with evidence suggesting that when flow information is particularly rich (Warren et al., 2001), or when alternative sources of

guidance level control. Looking to a distant point could provide additional useful information (e.g. retinal flow; Wann \& Swapp, 2000) but whether this is the case (and how the information is combined) has not been formally assessed. Although Kountouriotis et al. (2012) extended the Active Gaze Model to include road-edge information they did not set out to explicitly test two-level control (or manipulate flow information). 
Running Head: Optic Flow modulates Guidance Steering Control

information are weak (Wilkie \& Wann, 2002), retinal flow is important for steering control (for a fuller debate of whether retinal flow is used in the presence of other variables the reader is referred to Rushton et al., 1998; Fajen \& Warren, 2000; Harris \& Bonas, 2002; Harris \& Rodgers, 1999; Harris, 2001; Rushton \& Salvucci, 2001; Wann \& Land, 2000). Consistent with this argument, Li \& Chen (2010) have presented evidence that ground-flow information is used in addition to road-edge information in straight-path lane keeping. Kountouriotis et al. (2013) extended this finding to curvilinear bends. Kountouriotis et al. (2013) independently manipulated the textured regions outside and inside of a curved pathway to create asymmetric flow patterns, which led to systematic steering biases, despite the presence of visible road-edges. Kountouriotis, Mole, Merat, \& Wilkie (2016) showed similar systematic steering biases when global speed was varied, despite veridical (and sufficient) information from the demarcated road.

It seems, then, that humans steering curved trajectories are sensitive to global flow speed, and exhibit systematic directional steering biases depending whether flow speed is faster or slower than veridical (Kountouriotis et al., 2013, 2016). It remains unclear, however, how this use of flow fits with the two-level modes of guidance and compensatory control. To test the role of global flow in two-level control (Figure 1D), we manipulated near and far road-edge visibility alongside optic flow information. The pattern of steering biases across road-edge visibility conditions will reveal the extent to which flow contributes to driving. It is predicted that some flow bias will be observed in full road viewing conditions (as per Kountouriotis et al., 2013, 2016). However, it is unknown how flow bias will vary across different visibility conditions. Two mutually exclusive frameworks can be used to aid predictions of the pattern of results (see Figure $1 \& 2$ ): 
Running Head: Optic Flow modulates Guidance Steering Control

Modulation Hypothesis (H1): Flow modulates a control signal provided by road-edges

The Modulation Hypothesis is based on the assumption that the distribution of control processes across guidance and compensatory levels is determined by availability of taskrelevant information (road-edges). If guidance level (far road) information is unavailable, the control solution will be dominated by compensatory control; if compensatory level (near road) information is unavailable, the control solution will be dominated by guidance level control (Land \& Horwood, 1995). Within this framework the contribution of flow information is restricted to acting upon a road-edge signal (i.e. when a road-edge signal is 
absent, flow information is unable to contribute). This leads to three alternate hypotheses:

\section{H1. Modulation}

Hypothesis
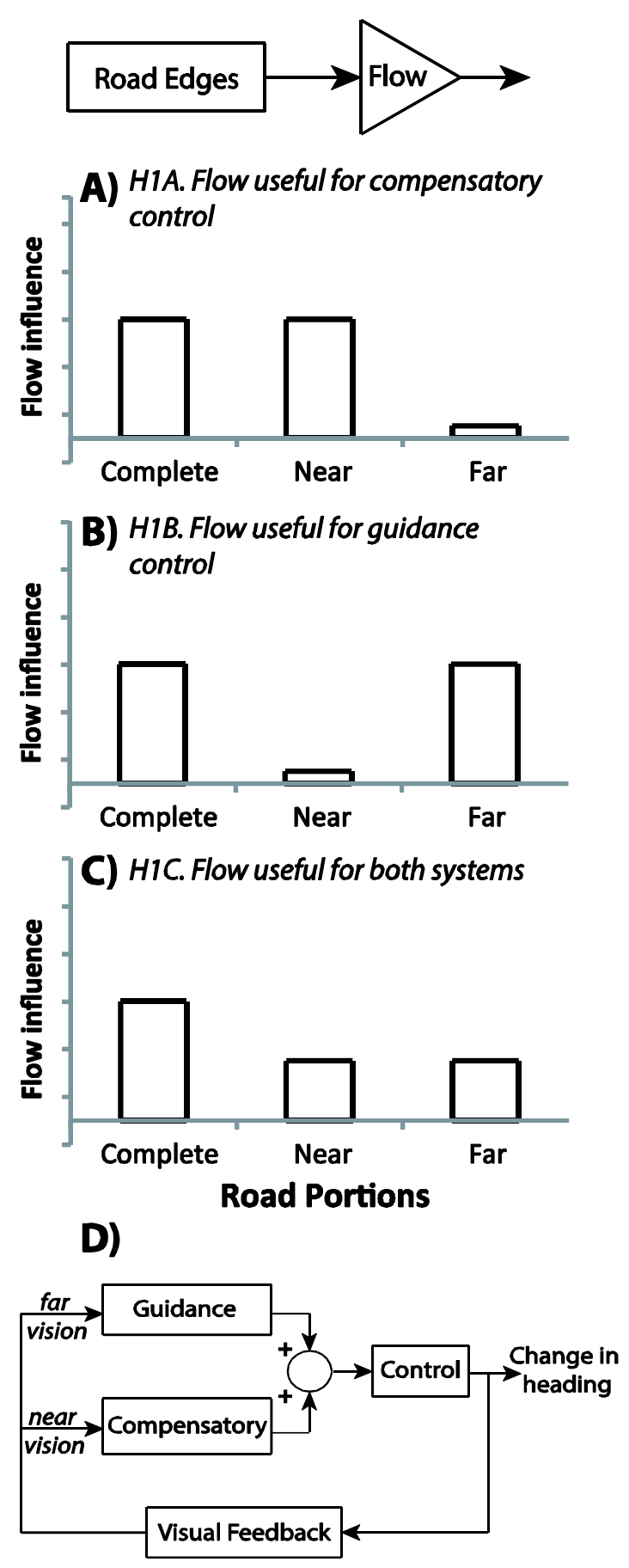

Figure 1. The Modulation Hypothesis proposes that flow modulates an existing control signal supplied by road-edges. AC) Possible patterns of results under the Modulation Hypothesis. We expect to observe some flow bias for full road conditions (as per Kountouriotis et al., 2013, 2016). Diminished flow influence when compensatory level road-edge information is removed (A) or guidance level road-edge information is removed (B) will indicate selective modulating of 
Running Head: Optic Flow modulates Guidance Steering Control

compensatory or guidance control respectively. C) A diminished (yet non-zero) flow influence across both Near and Far conditions will indicate that the flow influence observed in Kountouriotis et al. $(2013,2016)$ is dependent on both compensatory and guidance level road-edge information. D) The Two-level framework for steering control.

H1A) Flow modulates compensatory level control only: There is some evidence that, on curved paths, flow information is not useful for perceiving future path (Saunders \& Ma, 2011), but it can provide information about immediate heading (Warren, Mestre, Blackwell, \& Morris, 1991). It could be argued that instantaneous heading is not necessarily useful for specifying future steering requirements because it does not provide information about future path, but could be useful for compensatory level control in relation to the near road-edges. Removing near road-edges means that compensatory control is only possible through an uncertain estimate of where the near road-edges might be. Therefore, when near road-edge information is removed from the display, H1A would predict flow influence to diminish as guidance level control takes over (see Figure 1A).

H1B) Flow modulates guidance level control only: Whilst Saunders \& Ma (2011) contend that retinal flow does not support future circular path perception, there is some evidence that humans can gauge future path (from flow) adequately enough to steer (Cheng \& Li, 2011), and there are theoretical accounts that describe how the retinal flow pattern could be used to 'directly perceive' path (Kim \& Turvey, 1999; Wann \& Swapp, 2000). If flow contributes to estimating future path, H1B would predict an interaction between flow and far road-edge information. When far road-edge information is removed from the scene, the future steering requirements become uncertain, therefore the control solution would rely on compensatory control (near road-edges; Land \& Horwood, 1995) and flow influence will decrease (see Figure 1B). 
Running Head: Optic Flow modulates Guidance Steering Control

H1C) Flow ubiquitously modulates control: There is evidence that humans are able to judge future path from flow (Cheng \& Li, 2011; Kim \& Turvey, 1998; Wilkie \& Wann, 2006) so flow might contribute to guidance level control. However, there is also evidence that flow is useful for gauging instantaneous direction (Li, Chen, \& Peng, 2009; Li, Sweet, \& Stone, 2006), which could be useful for compensatory control. Additionally, Chatziastros et al. (1999) observed improved performance across all road viewing conditions when a strong flow signal was added (to a high contrast road surface). If flow is useful for both control systems flow should influence steering irrespective of which road components are available (Figure 1C).

\section{Weighted Combination Hypothesis (H2): Flow and road-edges provide independent}

\section{control signals that are flexibly combined}

The Modulation Hypothesis (H1) is inspired by Land and Horwood's (1995) observations that steering control strategies were largely provided by road-edge availability. However, Land \& Horwood's (1995) displays did not produce retinal flow. It is possible that retinal flow could provide information to supplement uncertain task information. In the Weighted Combination Hypothesis, flow and road-edge information are treated as two (potentially) competing sources of information that can be combined to provide inputs to a control solution (Figure 2; e.g. Wilkie \& Wann, 2002). In this case, when road-edge information is absent (effectively zero weight) the contribution of flow information would increase (effectively $100 \%$ weight). It is evident that road-edges are usually weighted highly by the visual system during curved trajectory driving (Kountouriotis et al., 2012), therefore when road-edge information is strong, and/or flow information weak, flow may only contribute little to steering control. Supporting this, Chatziastros et al. (1999) found that adding a weak flow signal (low resolution road texture) to black-background displays did not improve steering performance 
Running Head: Optic Flow modulates Guidance Steering Control

(complimentary results are reported by Kountouriotis \& Wilkie, 2013, for degraded dot flow displays). Using a strong retinal flow signal (large field-of-view, high resolution projector screen with global flow manipulations) Kountouriotis et al. $(2013 ; 2016)$ demonstrate a predictable amount of flow bias under full-road viewing conditions. H2 offers three competing hypotheses to predict the pattern of steering biases when different road-edge components are removed: 


\section{H2. Weighted Combination Hypothesis}
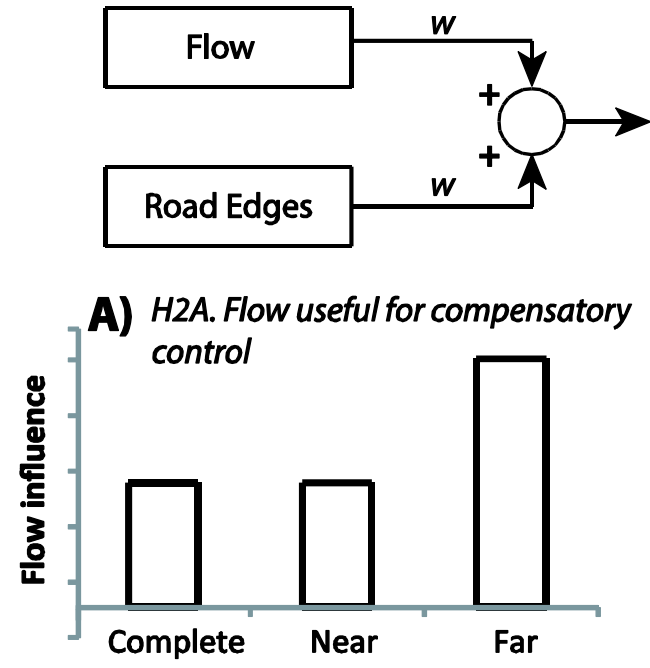

B) H2B. Flow useful for guidance control
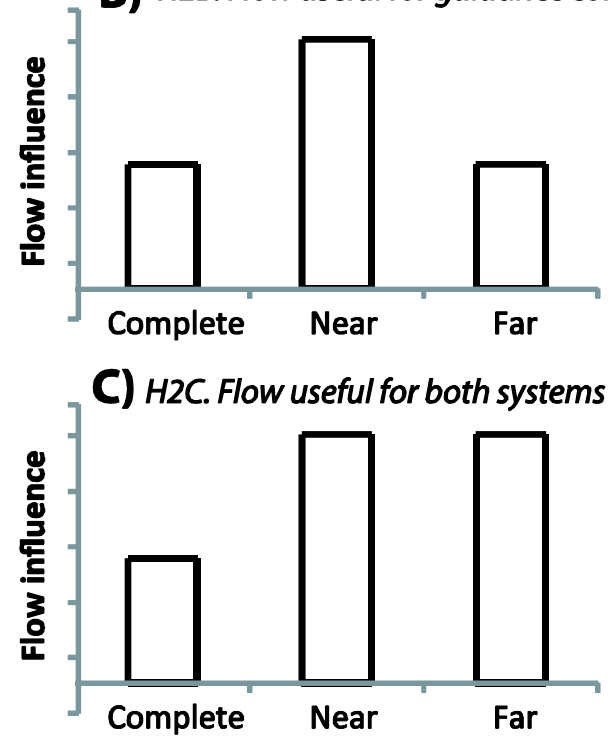

Road Portions

Figure 2. The Weighted Combination Hypothesis proposes that flow and road-edge information could be augmented in a weighted combinatory manner to provide a control solution. A) - C) possible patterns of results under the Weighted Combination Hypothesis. We expect to observe some flow bias for full road conditions (as per Kountouriotis et al., 2013, 2016). If flow information selectively interacts with compensatory or guidance level control, a weighted combination framework would predict an increase in flow influence when compensatory level road-edge information (A) or guidance level road-edge information (B) are removed, respectively.

$\mathrm{H} 2 \mathrm{~A}$ ) Flow is weighted and combined for compensatory level control only: If flow is treated as a weighted input to compensatory control, removing near road-edges will cause greater 
Running Head: Optic Flow modulates Guidance Steering Control

weight to be attributed to flow information. Therefore, flow influence will increase when near road-edge information is removed, but will remain unchanged when far information is removed (because the 'near only' condition provides the same compensatory control information as a complete road; Figure $2 \mathrm{~A}$ ).

$\mathrm{H} 2 \mathrm{~B})$ Flow is weighted and combined for guidance level control only: If flow is treated as a weighted input into guidance level control, removing far edges will cause more weight to be attributed to flow information. Therefore, flow influence will increase when far road-edge information is unavailable, but remain constant when near road-edge information is removed (Figure 2B).

$\mathrm{H} 2 \mathrm{C})$ Flow is ubiquitously weighted and combined for control: If flow is useful for both compensatory and guidance level control, flow will influence steering more whenever roadedge information is removed. This would be consistent with studies that demonstrate that human perceptual judgements rely upon the flexible weighted combination of information with weaker and less reliable signals being down-weighted (Ernst \& Banks, 2002). In this case one would expect to see a pattern similar to Figure $2 \mathrm{C}$.

\section{General Method}

To determine, specifically, the role of optic flow in providing information for guidance or stabilisation during steering, two experiments are reported. The first experiment examines whether optic flow interacts with road-edges by varying the presence of near or far road-edge information to see whether flow supports stabilising or guidance level steering control (whilst also examining the influence of gaze behaviours). The second experiment examines the relationship between road-edges and optic flow in greater detail by creating a wider set of 
Running Head: Optic Flow modulates Guidance Steering Control

road conditions (with each combination of near, middle and far road segments) as well as a number of different optic flow speeds (but with gaze constrained to track the centre of the road $1.2 \mathrm{~s}$ ahead). The common methodology used for both experiments is described below.

\section{Apparatus}

Using a driving simulator (Figure $3 \mathrm{~A}$ ), participants steered along a series of bends. Virtual environments were created using WorldViz Vizard 3.0 (WorldViz, Santa Barbara, CA) on a PC with Intel i7 $3770(3.40 \mathrm{GHz})$. Images were generated at $60 \mathrm{~Hz}$ and were back-projected using a Sanyo Liquid Crystal Projector (PLC-XU58, Sanyo, Watford, UK) onto a screen with dimensions of $1.98 \times 1.43 \mathrm{~m}$ in a matte-black viewing booth (the projector was the sole source of light). Images were perspective correct from a viewing distance of $1 \mathrm{~m}$ from the screen with eye-height of $1.2 \mathrm{~m}$ (total field of view $89.42^{\circ} \times 71.31^{\circ}$ ). Participants sat in a height-adjustable driving seat. Participants controlled steering using a force-feedback wheel (Logitech G27, Logitech, Fremont, CA) with self-centering torque. Steering wheel angle was linearly mapped onto rate of change of heading through a minimum step size of $.36^{\circ} / \mathrm{s}$ (i.e. as per previous publications, specific vehicle dynamics were not simulated so as to reduce the amount of practice needed to achieve stable performance, e.g. Cloete \& Wallis, 2011; Kountouriotis et al., 2012; Land \& Horwood, 1995; Robertshaw \& Wilkie, 2008; Wilkie \& Wann, 2002, 2003b).

In Experiment 1, eye-tracking was conducted using an ASL remote eye tracker to calibrate and record eye movements and a magnetic head tracker recorded head movements (Flock of Birds). The system was accurate to within $\pm .6^{\circ}$ (as per Kountouriotis et al., 2012; Wilkie, Kountouriotis, Merat, \& Wann, 2010). Participants' gaze was calibrated using a custom 9- 
Running Head: Optic Flow modulates Guidance Steering Control

point calibration procedure using WorldViz Vizard 3.0 (WorldViz, Santa Barbara, CA).

Recording of eye, head and steering data was synchronised with display update rate at $60 \mathrm{~Hz}$.

\section{Stimuli}

The simulated virtual environment consisted of a green tinted texture, with a $3 \mathrm{~m}$ wide road of constant curvature (60m radius) demarcated with white road-edges (as per Kountouriotis et al., 2012; see Figure 3B). The road had an initial straight section which was $6 \mathrm{~m}$ in length. In Constrained gaze conditions participants fixated a cross displayed $16.1 \mathrm{~m}(\sim 1.2 \mathrm{~s})$ ahead on the road centre (this controlled eye-movements which have been shown to be an important influence over steering in many situations). Participants were instructed to 'attempt to steer a central trajectory, keeping to the middle of the road'; to steer 'as smoothly and as accurately as you can'; and to 'centre the wheel after each trial'. Participants were asked to keep to the middle of the road so that any observed steering bias could be attributed to the flow speed manipulations and interpreted with respect to 'ideal' performance at the road centre (zero bias) ${ }^{3}$. Individual trials were 6 seconds long with a .83s (50 frame) pause at the start of trials to give participants time to re-centre the wheel before motion commenced: the driving time was therefore $5.17 \mathrm{~s}$. Only 270 frames were analysed $(4.5 \mathrm{~s}$ at $60 \mathrm{~Hz})$. Analysis starts from the end of the straight road section, which takes approximately $.45 \mathrm{~s}$ to travel ( 27 frames), and

\footnotetext{
${ }^{3}$ Instructing participants to maintain a central road position might be considered a limitation when trying to generalise the findings of the present research since 'corner cutting' behaviour is often observed when this instruction is omitted (Raw, Kountouriotis, Mon-Williams et al., 2012) and also in more naturalistic driving scenarios (Lappi, 2014). The purpose of corner-cutting is usually to reduce course curvature which allows smoother and/or potentially faster trajectories to be taken, however, corner cutting is not always exhibited by participants. For example on narrow roads when travelling at slow speeds less corner-cutting is exhibited, particularly by older adults (Raw et al., 2012). Given that without instruction individuals cut corners by different amounts, there was no single a priori 'ideal' line that could have been used to measure steering bias. In any case, it is unclear how the variation in steering biases observed across visual conditions could be explained by our instructions.
} 
the final 10 frames ( .17s) were also excluded from the analysis. There were six repeated trials of each condition.
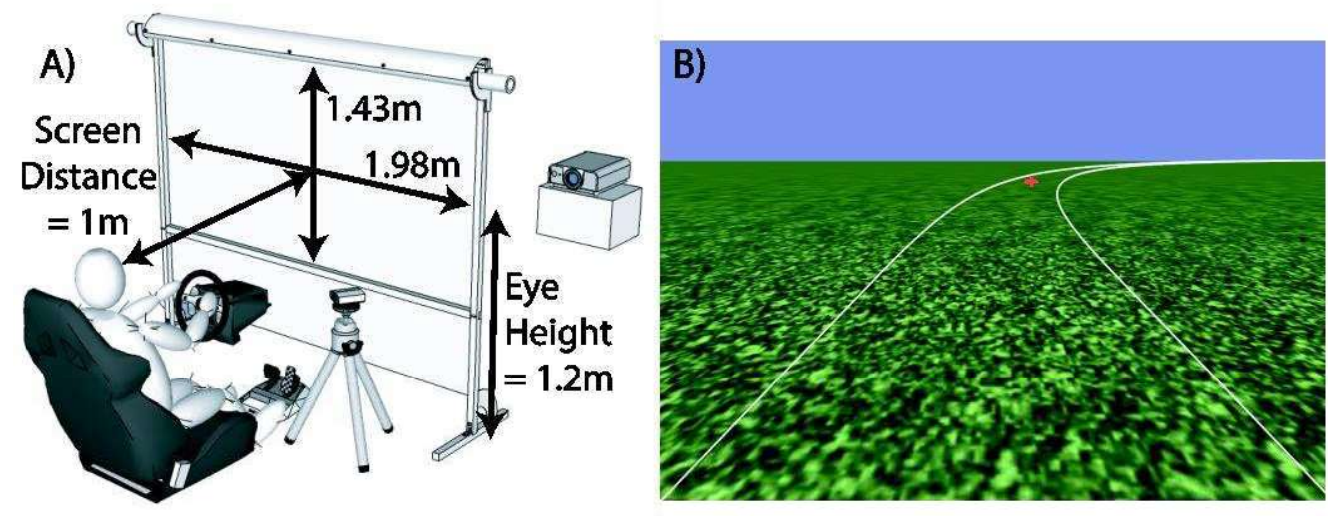

Figure 3. A) Schematic of Driving Simulator Layout. B) Screenshot of right-hand bend stimuli, with the red fixation cross used in Experiment 2 and in the 'Constrained gaze' condition of Experiment 1.

\section{Manipulating Flow}

A virtual environment with road-edges and ground texture has two sources of speed information: flow speed and rate of approach to road-edges. These two sources of information can be put into conflict (Figure 4). When the camera moves on a curved path the observer experiences counter-movement of texture elements which gives rise to the percept of self-motion. The same flow pattern can be simulated by keeping the camera stationary but rotating the ground-plane counter-clockwise (when steering a right-hand bend; Figure 4A). Using these principles, it is possible to produce a flow pattern indicative of any given velocity whilst keeping the actual driver velocity relative to road-edges constant (because flow vector length is an additive result of the driver velocity and ground-plane rotation; Figure 4B \& C). If the rate that the positional error develops is kept constant the requirements of the steering task (of keeping to the middle of the road) are the same across all flow conditions, therefore any steering bias across flow conditions can be attributed to the manipulation of global flow 
Running Head: Optic Flow modulates Guidance Steering Control

speed. In Experiment 1 and 2, the camera motion was always consistent with a locomotor speed of $13.41 \mathrm{~ms}$ ( $\sim 30 \mathrm{mph}$; when steering a constant curvature bend of $60 \mathrm{~m}$ radius this corresponds to an angular velocity of $12.8^{\circ} \%$ s).

\section{Camera Motion World Motion Displayed Flow}

A)
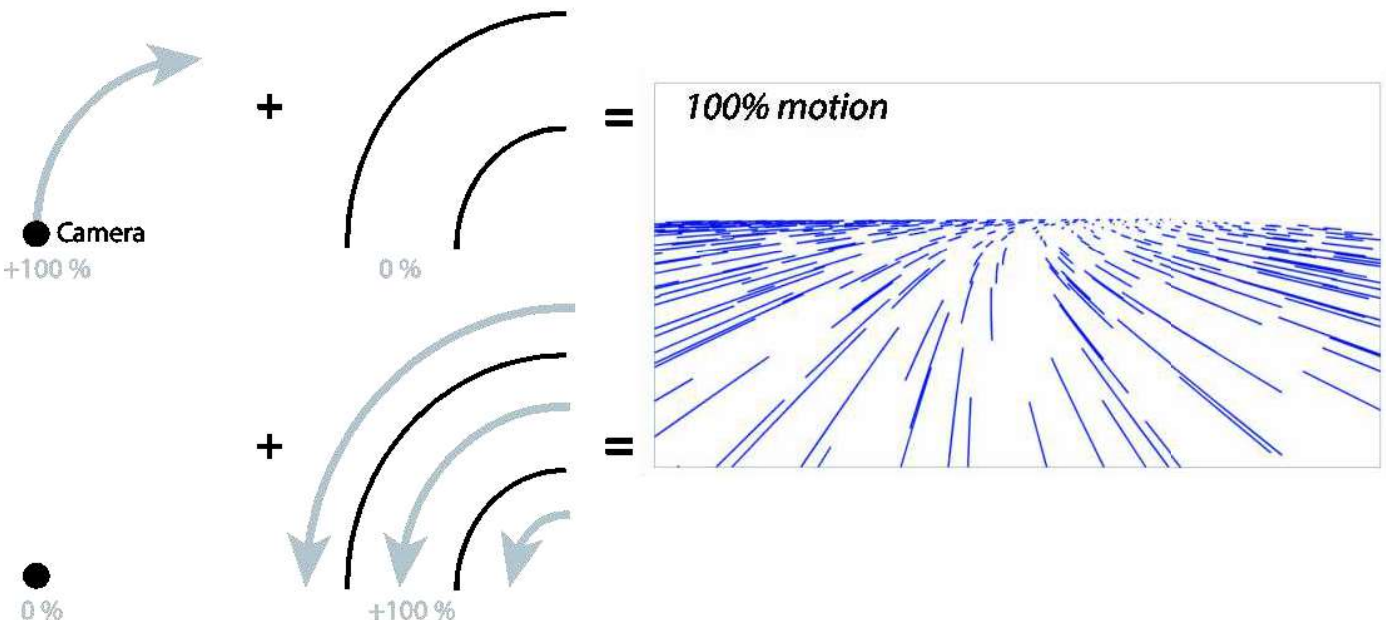

$0 \%$

$+100 \%$

B)
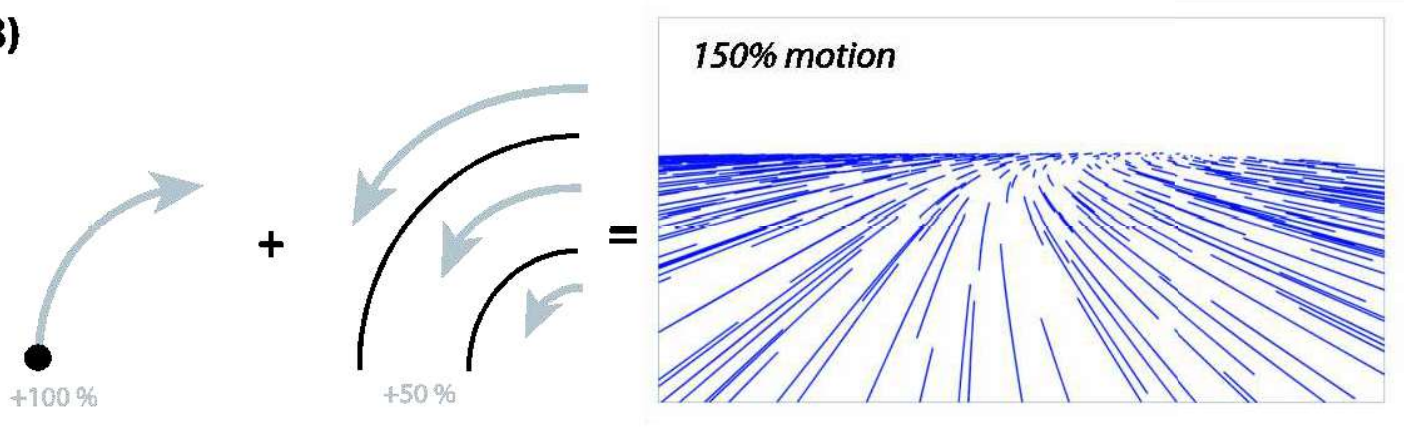

C)
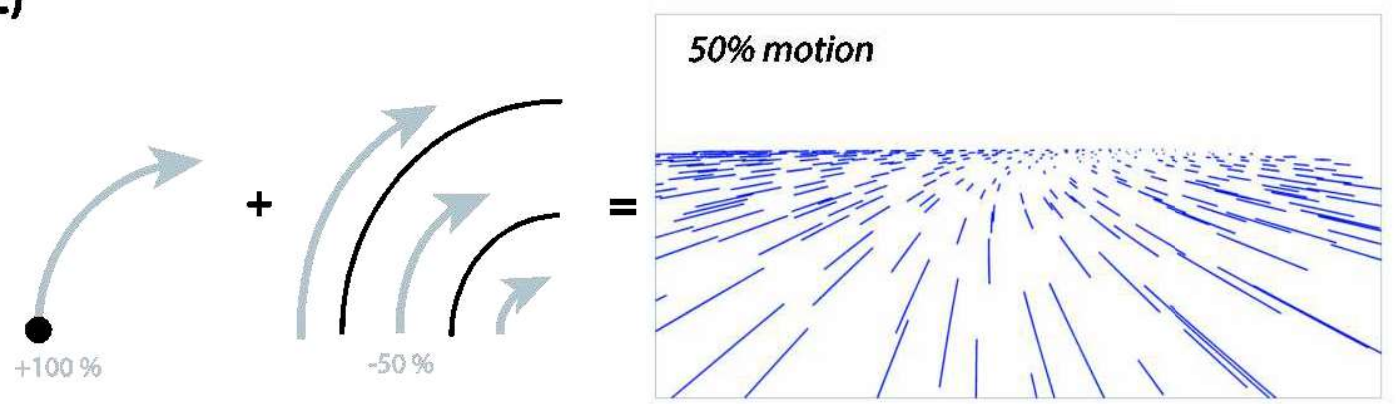

Figure 4. Schematic depicting methods of simulating perceived motion in a virtual environment. A) Given a trajectory of constant curvature, it is possible to generate identical flow patterns by either moving the camera or counter-rotating the ground plane. B) A faster flow can be generated by combining camera motion with ground plane counter rotation. The 
Running Head: Optic Flow modulates Guidance Steering Control

flow simulation corresponds to condition $\mathrm{FL}_{1.5}$ (Experiment 2). C) A slower flow pattern can be induced if the ground plane is rotated in the direction of camera movement (clockwise in right-hand bends). The flow simulation corresponds to condition $\mathrm{FL}_{.5}$ (Experiment 2). Flow simulations were generated over $.15 \mathrm{~ms}$, with the observer travelling a bend of $60 \mathrm{~m}$ radius at $13.41 \mathrm{~ms}$. Simulated gaze direction was at a fixation point $\sim 1.2 \mathrm{~s}$ ahead (as in Figure 3B).

\section{Manipulating Road-edges}

As per a number of previous studies, the amount of road-edge information was varied by explicitly rendering curvilinear markings that were super-imposed on top of the ground plane (Billington, Field, Wilkie, \& Wann, 2010; Cloete \& Wallis, 2011; Kountouriotis et al., 2012; Land \& Horwood, 1995; Neumann \& Deml, 2011; Wilkie et al., 2010). This method allows roadedge information to be manipulated without changing the underlying ground texture (hence the flow patterns are also unaffected).

In Experiment 1 and 2 the road was divided into three 'segments': 'Near' road (N), 'Mid' road (M), and 'Far' (F) road. For Experiment 1, these regions were respectively classified (as per Billington et al., 2010) as 0-6m (0-.45s) ahead, 6-12m (.45-.91s) ahead, and from $12 \mathrm{~m}$ up to the horizon. For Experiment 2, these regions were classified as $0-.5 \mathrm{~s}(6.71 \mathrm{~m})$ ahead, $.5 \mathrm{~s}-1 \mathrm{~s}$ $(13.41 \mathrm{~m})$ ahead, and $1 \mathrm{~s}$ to horizon. In these displays, the road up to .5s does not give any indication of future direction of travel (i.e. no curvature) but indicates immediate position relative to road-edges, whilst $\sim 1$ s to horizon allows preview for upcoming steering requirements but not information relative to immediate steering corrections. Additionally, $\sim$.5s and $\sim 1 s$ ahead are similar to the near and far optima reported in Land (1998). Figure 5 shows all road conditions present in Experiment 2 (a subset of these are used in Experiment 1). 

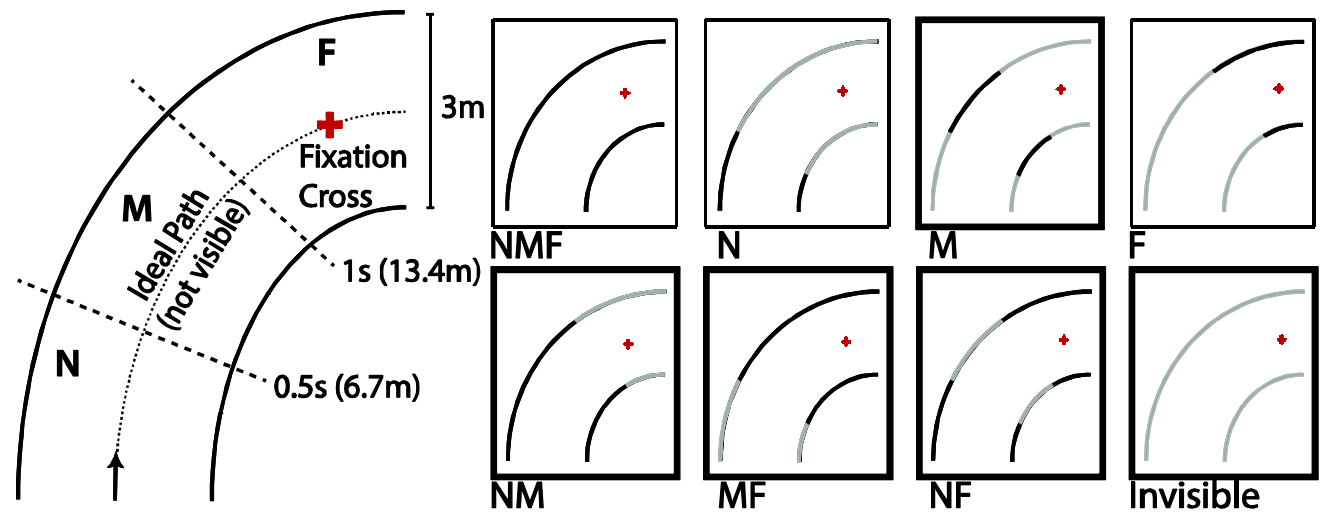

Figure 5. Birds-eye schematic of all road conditions present in Experiment 2. Road display conditions are denoted by their constituent parts. $\mathrm{N}=$ road up to $.5 \mathrm{~s}$ ahead, $\mathrm{M}=$ road from $.5 \mathrm{~s}$ to $1 \mathrm{~s}$ ahead, $\mathrm{F}=$ road from $1 \mathrm{~s}$ to horizon. The conditions of M, NM, MF, NF, and Invisible (emboldened boxes) were not present in Experiment 1, but were included in Experiment 2.

\section{Experiment 1: Unconstrained \& Constrained Gaze}

The primary purpose of this experiment was to determine whether the influence of flow upon steering varied according to whether near or far road-edge information was available. The frameworks highlighted in $\mathrm{H} 1 \mathrm{~A}-\mathrm{C}$ (Figure 1) and $\mathrm{H} 2 \mathrm{~A}-\mathrm{C}$ (Figure 2) will be used to determine how flow is used when steering across a range of road conditions. It is, however, also important to consider the possible influence of eye movements when steering (Wilkie et al., 2010), since road-edge information may not be the sole determinant of flow use. Gaze behaviours can interact with the use of road-edge or flow information (Kountouriotis et al., 2012), and where a driver looks in the scene may affect how they utilise flow (Authié \& Mestre, 2012). Gaze patterns are heavily influenced by steering requirements (Wilkie et al., 2008), and steering behaviour can in turn be biased by gaze patterns (Wilkie et al., 2010). Specifically, gaze distributions have been shown to be influenced by road-edge availability: fading or removing one road-edge causes horizontal relocation of gaze toward the remaining road-edge (Kountouriotis et al., 2012). It might be predicted, therefore, that removing near 
Running Head: Optic Flow modulates Guidance Steering Control

or far road segments will cause predominantly vertical gaze shifts towards the visible edges. Such shifts will change the available extra-retinal information that is often used as a guidance signal (Salvucci \& Gray, 2004; Wilkie \& Wann, 2005), as well as changing the retinal flow patterns (e.g. Wann \& Swapp, 2000). Unfortunately, most studies that manipulate near and far road-edge information do not record eye-movements (e.g. Chatziastros et al., 1999; Cloete \& Wallis, 2011; Frissen \& Mars, 2014; Neumann \& Deml, 2011; van Leeuwen, Happee, \& de Winter, 2014), or do not report gaze behaviour when road-edges are removed (Land \& Horwood, 1995). The effect of removing near or far road-edge information upon gaze behaviour will be examined in this experiment to document how gaze patterns change when road-edges are manipulated.

For the reasons outlined above (and as per Robertshaw \& Wilkie, 2008) participants initially observed freely the stimuli when steering (Unconstrained gaze); they then repeated the experiment with gaze constrained to look at a standardised fixation point (Constrained gaze).

\section{Methods}

\section{Participants}

A sample of 13 University students (9 females, 4 males, ages 20 to 31, mean 24.7 years), were recruited. All had normal or corrected-to-normal vision, and all held a full driving license (average time since driving test $=5.7$ years). All participants were naïve as to the purpose of the experiment. All participants gave written informed consent and the study was approved by the University of Leeds Research Ethics Committee (Ref: 13-0221), and complied with all guidelines set out in the declaration of Helsinki.

\section{Procedure}


Running Head: Optic Flow modulates Guidance Steering Control

After calibration participants completed 8 practice trials (veridical flow speed, each road visibility condition, for left and right bends) to familiarise themselves with the simulator dynamics. There were three flow manipulations: slower-than-veridical (8.9ms; $20 \mathrm{mph})$, veridical (13.41ms; $\sim 30 \mathrm{mph}$ ) and faster-than-veridical (17.9ms; $\sim 40 \mathrm{mph})$, abbreviated to $\mathrm{SL}_{\mathrm{F}}$ ('SLower Flow'), VEF ('VEridical Flow'), and FS $\mathrm{F}_{\mathrm{F}}$ ('FaSter Flow'). There were also three road manipulations: 'Complete Road', 'Near Road', and 'Far Road', abbreviated to $\mathrm{NMF}_{\mathrm{Rd}}, \mathrm{N}_{\mathrm{Rd}}$, and $F_{\text {Rd }}$ (Figure 5). As per Robertshaw \& Wilkie, (2008) and Wilkie et al. (2010) we minimised the influence of the experimental setup on participant's natural gaze sampling behaviour by testing Unconstrained gaze conditions first, followed by the Constrained gaze conditions. Counterbalancing the conditions would have led to some Unconstrained gaze conditions being tested after Constrained gaze conditions which may have resulted in participants gaze patterns to be biased by the prior exposure to the fixation task. 
Running Head: Optic Flow modulates Guidance Steering Control

\section{Analysis}

Steering behaviour was captured by quantifying positional error with reference to the ideal path (specified as the lane centre). As per Wilkie \& Wann (2003b; see also Kountouriotis et al., 2012; Robertshaw \& Wilkie, 2008), Steering Bias and Root-Mean-Squared Error (Steering Error) were calculated to capture important aspects of steering behaviour within each trial with respect to the instruction 'stay as close to the centre of the road as possible'. Since participants were also asked to steer 'as smoothly and as accurately as they can', a measure of smoothness was also recorded, using the rate of change of movement of the steering wheel (Steering Wheel Jerk).

It was observed post-experiment that the method of continuously updating the far roadedges caused the update rate (which was synchronised with data recording rate) to reduce to

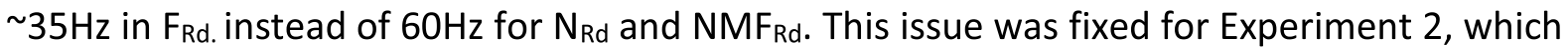
had a consistent update rate of $60 \mathrm{~Hz}$ across all conditions. The authors believe that this may have contributed to increased jerk values for $F_{R d}$ for Experiment 1 (as is highlighted in footnote 6), but are confident upon comparison with Experiment 2 that no other measures were compromised.

\section{Steering Bias (SB)}

Steering bias (Constant Error) was calculated to measure positional deviation from the lane centre for every frame, providing a signed directional measure of error (whereby positive bias denotes 'oversteering' and negative bias denotes 'understeering') ${ }^{4}$. The findings from

\footnotetext{
${ }^{4}$ This manuscript uses the terms 'oversteering' and 'understeering' to denote a participant steering towards the inside road-edge and outside road-edge respectively. These terms are distinct from the terms 'oversteer' and 'understeer' used to describe the response of real vehicles as per ISO 8855.
} 
Running Head: Optic Flow modulates Guidance Steering Control

Kountouriotis et al. (2016) suggest that under full road view conditions systematic changes in directional error occur when flow speed is manipulated. SB, then, will be the primary measure of interest. The extent that systematic shifts in SB are observed under specific road conditions will indicate whether flow is being used for steering control.

\section{Steering Error (RMSE)}

Root-Mean Squared Error (RMSE) was calculated from the magnitude of positional error (relative to the lane centre) at every frame. This measured both deviation and consistency of steering (but not systematic directional error). It would be predicted that RMSE should reduce when near road components are available, and increase when only far road-edge information is available (Land \& Horwood, 1995; Salvucci \& Gray, 2004).

\section{Steering Smoothness (SWJ)}

Steering Wheel Jerk (deg. $\mathrm{s}^{-3}$; SWJ) is the change in angular acceleration of the steering wheel, and can be used to indicate steering smoothness. Because a fixed position of the steering wheel causes a change in heading angle (i.e. an angular velocity), angular jerk reflects accelerated turning of the steering wheel. Large jerk values will, therefore, correspond to rapid steering corrections. It can be seen that this measure does not explicitly relate to the requirement to maintain a position near the road midline so it is possible to steer very smoothly but completely erroneously. The literature that has examined near and far road components suggests that smoother steering should be observed when far road components are available, and less smooth when only near road-edge information is available (Cloete \& Wallis, 2011; Frissen \& Mars, 2014; Land \& Horwood, 1995; Salvucci \& Gray, 2004).

\section{Flow Induced Steering Bias (FISB)}


Running Head: Optic Flow modulates Guidance Steering Control

Experiment 1 set out to examine whether the influence of flow varied depending on road component availability. Results from Kountouriotis et al. (2016) suggest that (under complete road conditions) the observed change in steering bias will be proportional to the changes in flow speed. Therefore, the gradient of a line fitted between $S B$ estimates in $S L_{F}, V E_{F}$, and $F S_{F}$, indicates how heavily a driver relies on flow: this measure will be referred to as Flow-InducedSteering-Bias (FISB). Calculating the gradient was simply approximated as: $\left(\mathrm{FS}_{\mathrm{F}}-\mathrm{SL}_{\mathrm{F}}\right) / 2$. The greater the FISB score, the more sensitive steering was to the flow manipulations in the predicted direction (oversteering in $\mathrm{FS}_{\mathrm{F}}$, understeering in $\mathrm{SL}_{\mathrm{F}}$ ). A negative FISB score would indicate that steering occurred in the opposite direction to predictions, and a near zero FISB score would indicate that steering was largely unaffected by the flow manipulations.

\section{Quantifying gaze behaviour: Lookahead Distance, Angular Road Offset, and Focal Area}

During the experiment the head was unconstrained to allow natural gaze behaviours. The average head rotation during trials was around $\pm 1^{\circ}$. There are many ways to analyse gaze data, but the focus of this experiment was to determine whether different conditions caused gaze distribution to vary vertically, horizontally, or to be more or less concentrated within a particular region. Therefore, the following metrics were calculated: Lookahead Distance, Angular Road Offset, and Focal Area (Figure 8B).

Eye movement recordings of gaze coordinates for each trial were placed into spatial bins of $.89^{\circ} \times .71^{\circ}(100 \times 100$ bins $)$. A 2 D Gaussian low pass filter of size $10 \times 10$ and $\sigma=.3$ was then applied to these bins for smoothing. The bin of highest fixation density was identified per trial, from this point the Lookahead Distance (LD; depth in metres) and the Angular Road Offset (ARO; signed angular deviation from a point on the road centre at the same lookahead distance as the bin of highest fixation density) was calculated. The bin of highest fixation 
Running Head: Optic Flow modulates Guidance Steering Control

density was used instead of the average point because averaging across all the data makes the point estimate susceptible to bias from occasional eccentric fixations or noise in the eyemovement signal (e.g. blinks). Finally, Focal Area (FA) was calculated as a measure of gaze concentration. The binned data was used to determine the contoured region around the point of highest density where $25 \%$ of gaze fixation points fell (Figure $8 \mathrm{~B}$ ). The size of this area (in degrees $^{2}$ ) provided a measure of gaze concentration.

\section{Results: Steering Behaviour}

A 2 (Gaze: Unconstrained, Constrained) $\times 3$ (Road: $N_{M} F_{R d}, N_{R d}, F_{R d}$ ) repeated measures ANOVA was conducted on FISB and a 2 (Gaze: Unconstrained, Constrained) $\times 3$ (Flow: VEF, $\left.S L_{F}, F S_{F}\right) \times 3$ (Road: $N_{M F_{R d}}, N_{R d}, F_{R d}$ ) repeated measures ANOVA was conducted on SB, RMSE and SWJ measures. Greenhouse-Geisser corrections are reported when assumptions of sphericity were violated. Of particular interest was how steering behaviour in manipulated conditions compared to behaviour in the control condition, where full road-edge information was available and flow speed was veridical. Therefore planned simple contrasts compared each level to the control condition $\left(\mathrm{VE}_{\mathrm{F}}+\mathrm{NMF}_{\mathrm{Rd}}\right)$. Partial eta squared, $95 \%$ confidence intervals and standard error of the mean (SEM) are reported where appropriate.

\section{Flow-Induced Steering Bias (FISB)}

FISB magnitude highlights the extent to which flow influenced steering across road conditions. Figure $6 A \& B$ shows that participants exhibited large positive FISB in $\mathrm{NMF}_{R d}$ and $\mathrm{F}_{\mathrm{Rd}}$, but not in $\mathrm{N}_{\mathrm{Rd}}$ trials. A 2 (Gaze) $\times 3$ (Road) repeated measures ANOVA (Table 1) revealed a significant effect of Road, but no effect of Gaze, nor any interaction. Planned contrasts showed that for both gaze conditions FISB for $\mathrm{N}_{\mathrm{Rd}}$ were significantly less than $\operatorname{NMF}_{\mathrm{Rd}}(F(1,12)$ 
Running Head: Optic Flow modulates Guidance Steering Control

$\left.=24.02, p<.001, \eta_{p}^{2}=.67\right)$, whereas $F_{R d}$ did not significantly differ from $\operatorname{NMF}_{R d}(F(1,12)=$ $\left..72, p=.41, \eta_{p}^{2}=.057\right)$. It seems that steering behaviour was most affected by flow speed manipulations when guidance (far road) information was present (as in the case of $F_{R d}$ and $\mathrm{NMF}_{\mathrm{Rd}}$ ) and least affected when guidance information was not available.

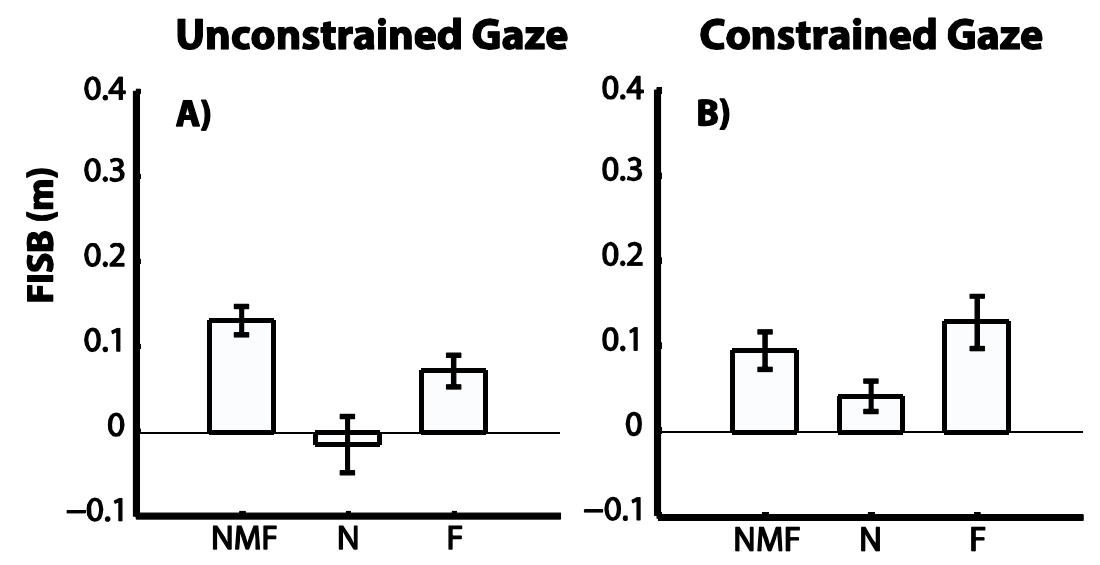

Figure 6. FISB for Experiment 1 for Unconstrained gaze (A) and Constrained gaze (B). Error bars represent standard error of the mean.

\section{Steering Bias (SB)}

FISB is a useful measure to simply examine the extent to which participants responded to flow speed under the different road-edge conditions. There are, however, different patterns of steering bias that could lead to similar FISB scores. As can be seen from Figure 7A \& $B$, the flow manipulations generally produced predictable steering biases in both Unconstrained and Constrained gaze conditions: faster flow produced oversteering, and slower flow produced understeering. However, this pattern did vary to some extent across road and gaze conditions. The full ANOVA results are displayed in Table 1. There were main effects for all three factors, as well as a Gaze $\times$ Road interaction and a Flow $\times$ Road interaction. The Gaze $\times$ Road interaction was caused by levels of understeering increasing when gaze was unconstrained for $\mathrm{N}_{\mathrm{Rd}}$, compared to $\operatorname{NMF}_{\mathrm{Rd}}\left(F(1,12)=30.91, p<.001, \eta_{\mathrm{p}}{ }^{2}=.72\right)$. The Flow $\times$ 
Running Head: Optic Flow modulates Guidance Steering Control

Road interaction was caused by the difference between $\mathrm{VE}_{\mathrm{F}}$ and both $\mathrm{SL}_{\mathrm{F}}(F(1,12)=6.49, p=$ $\left..026, \eta_{\mathrm{p}}^{2}=.35\right)$ or $\mathrm{FS}_{\mathrm{F}}\left(F(1,12)=9.84, p=.009, \eta_{\mathrm{p}}^{2}=.45\right)$ diminishing in $\mathrm{N}_{\mathrm{Rd}}$, compared to $\mathrm{NMF}_{\mathrm{Rd}}$. These results suggest that during Unconstrained $\mathrm{N}_{\mathrm{Rd}}$ participants exhibited more understeering and were less biased by flow manipulations than during Constrained $\mathrm{N}_{\mathrm{Rd}}$, but for $F_{R d}$ and $N M F_{R d}$ steering bias was similar across both gaze conditions (see Figure 7A \& $B$ ). 
Unconstrained Gaze Constrained Gaze
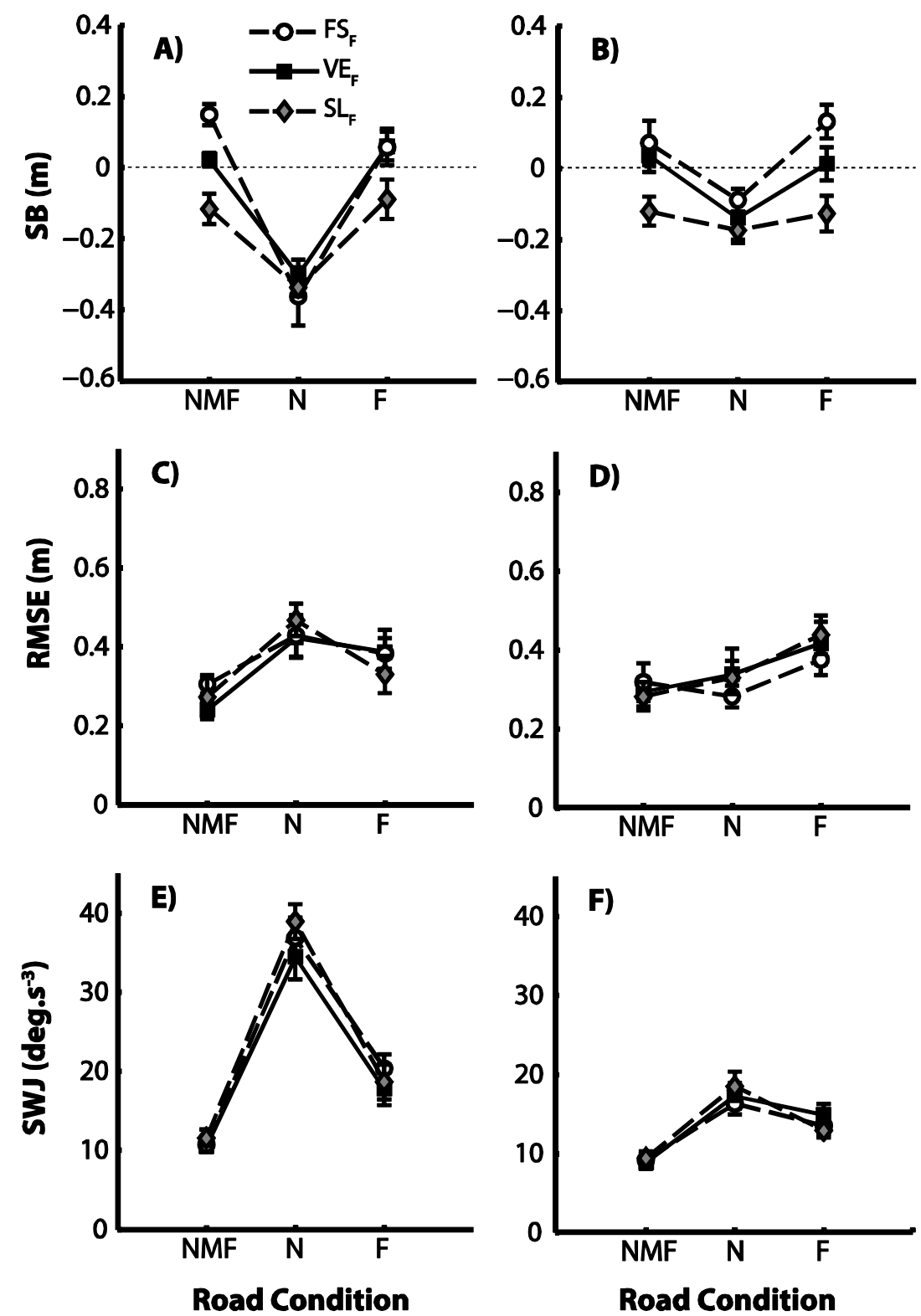

Figure 7. Steering performance measures across $\mathrm{FS}_{\mathrm{F}}$ (empty circles, dashed line), $\mathrm{VE}_{\mathrm{F}}$ (filled squares, solid line), and $\mathrm{SL}_{\mathrm{F}}$ (grey diamonds, dashed line), showing A-B) Steering Bias, C-D) Steering Error, and E-F) Steering Smoothness, for Unconstrained (left) and Constrained (right) gaze conditions. Error bars represent standard error of the mean (SEM).

\section{Steering Error (RMSE)}

Steering errors did not seem to systematically vary across flow levels (Figure 7C \& D). However, there were clear differences in RMSE across road visibility conditions. Steering errors were smallest when the complete road was present, but equivalent for $N_{R d}(M=.38 m$, 
Running Head: Optic Flow modulates Guidance Steering Control

$[.29, .47], \mathrm{SEM}=.042)$ and $\mathrm{F}_{\mathrm{Rd}}(\mathrm{M}=.39 \mathrm{~m},[.3, .48], \mathrm{SEM}=.041)$ conditions. Also of note is the larger RMSE under $\mathrm{N}_{\mathrm{Rd}}$ conditions when gaze was unconstrained $(\mathrm{M}=.44 \mathrm{~m},[.34, .54]$, $\mathrm{SEM}=$ $.046)$ and substantially smaller RMSE when gaze was constrained ( $M=.32 \mathrm{~m},[.23, .4]$, SEM $=$ .041). The two other road conditions did not change dramatically across gaze conditions. The ANOVA (see Table 1) reveals a main effect of Road and a Gaze $\times$ Road interaction. The interaction was caused by the RMSE score for $\mathrm{N}_{\mathrm{Rd}}$ reducing when gaze was constrained, whereas $\mathrm{NMF}_{\mathrm{Rd}}$ remained constant $\left(F(1,12)=20.81, p=.001, \eta_{\mathrm{p}}^{2}=.63\right)$.

\section{Steering Smoothness (SWJ)}

There are some clear differences in SWJ between gaze and road conditions (Figure 7E \& F). Across both gaze conditions, $\mathrm{N}_{\mathrm{Rd}}$ is the most jerky, followed by $\mathrm{F}_{\mathrm{Rd}}$ then $\mathrm{NMF}_{\mathrm{Rd}}$. SWJ was higher for $N_{R d}$ when participants had unconstrained gaze $\left(M=36.75\right.$ deg. $s^{-3},[31.66,41.83]$, $\mathrm{SEM}=2.33)$ compared to when eye movements were constrained $\left(M=13.52 \mathrm{deg} . \mathrm{s}^{-3},[11.29\right.$, $15.75]$, SEM = 1.02). The same trend is true for the other road visibility conditions, although less pronounced.

The ANOVA (see Table 1) shows that these differences resulted in main effects for Gaze and Road (and very large effect sizes for both), and interactions between Gaze $\times$ Flow and Gaze $\times$ Road. Contrasts showed the nature of the Gaze $\times$ Flow interaction was that the discrepancy between $\mathrm{SL}_{\mathrm{F}}$ and $\mathrm{VE}_{\mathrm{F}}$ was significantly larger for Unconstrained gaze than for Constrained gaze $\left(F(1,12)=5.20, p=.042, \eta_{p}^{2}=.30\right)$. The same pattern was observed for $\mathrm{FS}_{F}$, although the contrast did not quite reach significance $\left(F(1,12)=4.46, p=.056, \eta_{p}^{2}=.27\right)$. The Gaze $\times$ Road interaction was primarily driven by the difference between $\mathrm{N}_{\mathrm{Rd}}$ and $\mathrm{NMF}_{\mathrm{Rd}}$ reducing from Unconstrained to Constrained gaze $\left(F(1,12)=61.43, p<.001, \eta_{p}{ }^{2}=.95\right)$. A similar, but smaller, discrepancy reduction also happens for $\mathrm{F}_{\mathrm{Rd}}\left(F(1,12)=5.028, p=.045, \mathrm{\eta}_{\mathrm{p}}{ }^{2}=.30\right)$. To summarise, 
Running Head: Optic Flow modulates Guidance Steering Control

steering became jerkier when road-edge segments were removed, especially guidance information, and became less jerky when gaze is constrained to a point on the future path, designated by the fixation cross. Additionally, when gaze was stabilised SWJ was unaffected by the flow speed manipulation. 


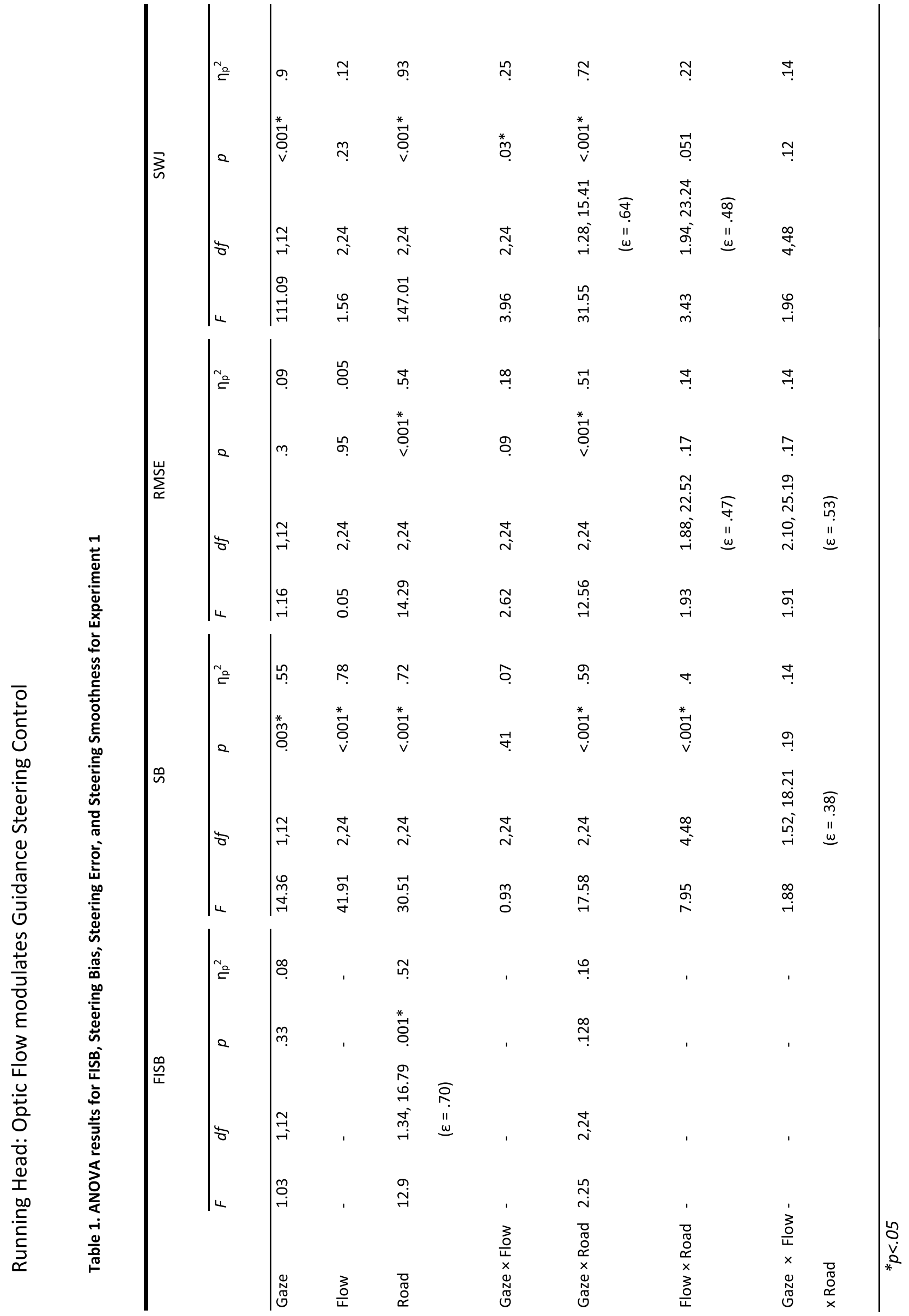


Running Head: Optic Flow modulates Guidance Steering Control

\section{Results: Gaze Behaviour}

Eye tracking data was successfully obtained for 9 out of the 13 participants ${ }^{5}$. In the second part of Experiment 1 participants were asked to look at a fixation cross displayed on the future path (Constrained gaze). We expected participants to follow these instructions so did not predict any gaze differences across visual conditions when fixating the cross. This was confirmed with no significant differences across conditions for any of the three measures (LD, ARO, and FA). To examine unconstrained gaze patterns (from the first part of Experiment 1), a 3 (Flow) $\times 3$ (Road) repeated measures ANOVA was conducted for each gaze measure in Unconstrained gaze conditions. As with the steering measures, significant differences were examined further using planned contrasts to compare every level to the control condition $\left(V_{F}+N M F_{R d}\right)$.

\section{Binned Data}

The individual gaze data, from which LD, ARO, and FA were calculated, were pooled across all participants and are displayed as isodensity contours in Figure 8A. Under complete road conditions $\left(\mathrm{NMF}_{\mathrm{Rd}}\right)$, gaze was concentrated in a region $10-20 \mathrm{~m}$ ahead, in the centre of the road. Equivalent gaze distributions were observed for $F_{R d}$. However, when far road-edge information was removed, gaze was 'pulled' downwards towards the near road-edges and was dispersed more widely, with no concentrated high density focal point. This pattern is reflected in the averaged group metrics displayed in Figure 9. The LD is closer (Figure 9A), and the Focal Area larger (Figure $9 \mathrm{C}$ ), for $\mathrm{N}_{\mathrm{Rd}}$ than either $\mathrm{NMF}_{\mathrm{Rd}}$ or $\mathrm{F}_{\mathrm{Rd}}$.

\footnotetext{
${ }^{5}$ Three of the participants wore glasses, making it difficult for the eye tracking to pick up the pupil and corneal reflex; and gaze data for a fourth participant was excluded because head tracking data failed to record.
} 
Running Head: Optic Flow modulates Guidance Steering Control

Changes in Flow speed did not seem to cause large changes in gaze patterns, demonstrated by similar pooled distribution maps across flow levels (Figure 8A). However, the group measures (Figure 9) show there may be subtle gaze behaviours that are difficult to pick out from the pooled distribution maps; for instance $F_{R d}$ and $N_{M} F_{R d}$ may not be equivalent across all measures, and there may have been consistent differences between Flow conditions in ARO and FA (Figure 9B \& C). 


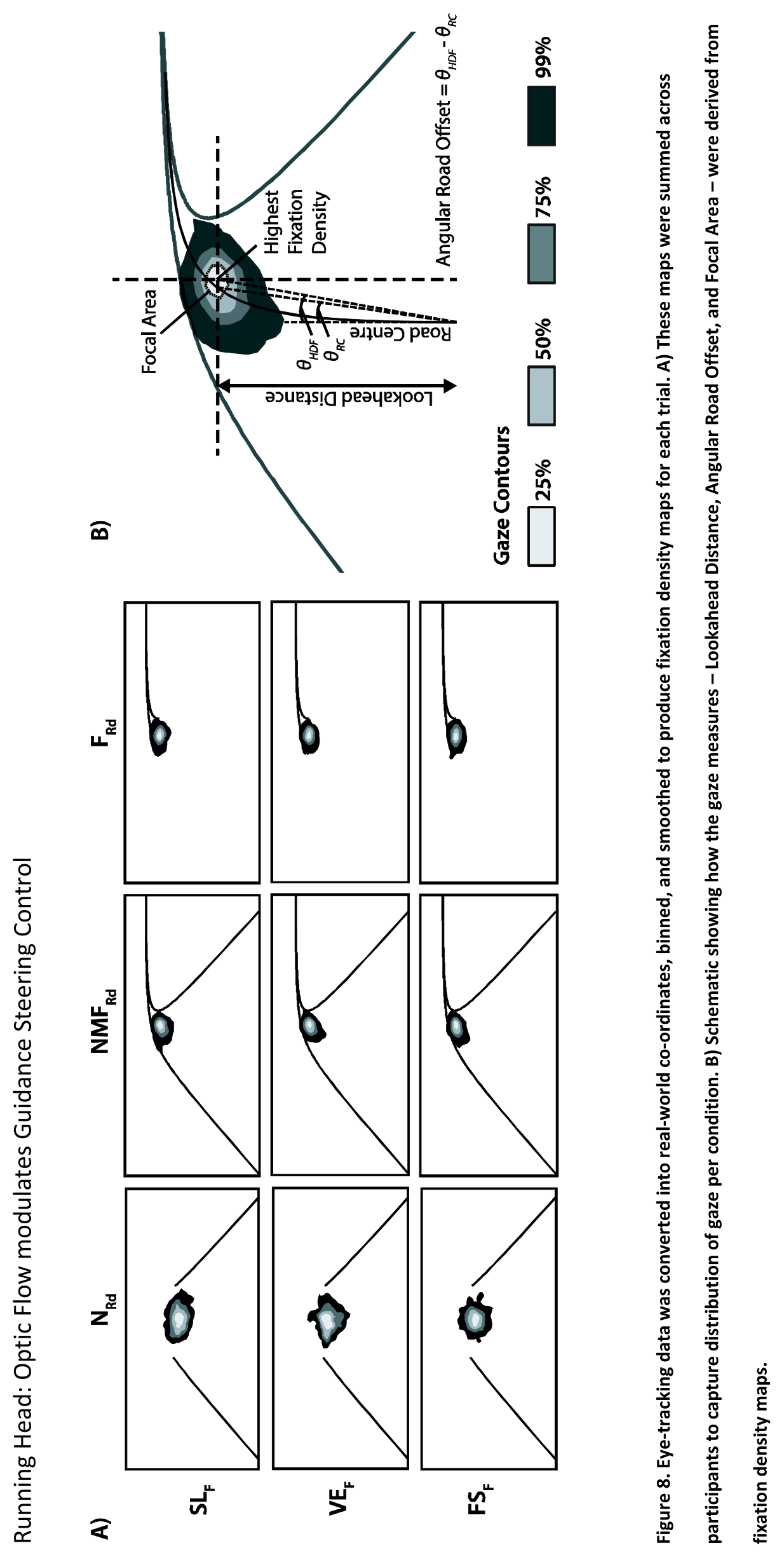


Running Head: Optic Flow modulates Guidance Steering Control

\section{Lookahead Distance (LD)}

As can be seen from Figure 9A, LD varied with road-edges. Participants looked lower in the scene when far road-edge information was omitted and higher in the scene during $\mathrm{NMF}_{\mathrm{Rd}}$ and $F_{R d}$ conditions. The ANOVA (see Table 2) revealed there to be main effects of Road and Flow, but no interaction. The main effect of road was due to a large reduction in LD from $\mathrm{NMF}_{\mathrm{Rd}}$ to $\mathrm{N}_{\mathrm{Rd}}\left(F(1,8)=188.8, p<.001, \eta_{\mathrm{p}}^{2}=.96\right)$. The main effect of flow was due to participants looking slight lower during $S L_{F}$, compared to $\operatorname{VE}_{F}\left(F(1,8)=7.43, p=.026, \eta_{p}{ }^{2}=.48\right)$; in practical terms, however, this difference amounts to less than .03s. 

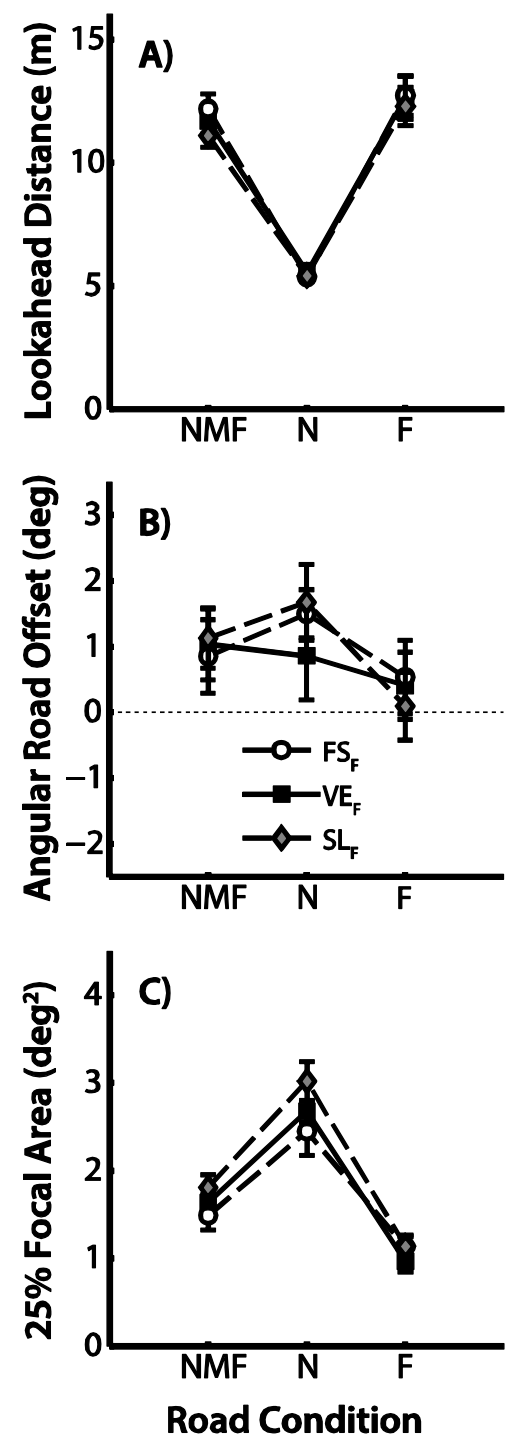

Figure 9. All gaze performance measures for Unconstrained gaze conditions across $F S_{F}$ (empty circles, dashed line), $V E_{F}$ (filled squares, solid line), and $\mathrm{SL}_{\mathrm{F}}$ (grey diamonds, dashed line), showing A) Lookahead Distance, B) Angular Road Offset (Gaze Offset from the Road Centre), and C) Focal Area where $25 \%$ of Gaze falls. Error bars represent standard error of the mean (SEM).

\section{Angular Road Offset (ARO)}

This measure assessed how close to the centre of the road people looked. Figure 9B shows that generally participants looked very close to the centre of the road (within $2^{\circ}$ ) even though gaze was unconstrained and there was no explicit visual marker for this location. The grand 
Running Head: Optic Flow modulates Guidance Steering Control

mean was under $1^{\circ}$ away from the road centre $\left(M=.9^{\circ},[-.017,1.82]\right.$, SEM $\left.=.4\right)$. The ANOVA (see Table 2) revealed a significant effect of road on ARO but no significant effect of Flow, nor any interaction. Contrasts revealed that participants looked closer to the centre (their ARO was reduced) in $\mathrm{F}_{\mathrm{Rd}}$ compared to $\operatorname{NMF}_{\mathrm{Rd}}\left(F(1,8)=7.19, p=.028, \eta_{\mathrm{p}}^{2}=.47\right)$. However, the difference between $\mathrm{F}_{\mathrm{Rd}}$ and $\mathrm{NMF}_{\mathrm{Rd}}$ was only $.66^{\circ}[-1.23,-.093]$, which at $12 \mathrm{~m}$ (the combined mean $L D$ for $F_{R d}$ and $N F_{R d}$ is $12.1 \mathrm{~m}$ ) equates to only a $10 \mathrm{~cm}$ difference on the road.

\section{Focal Area (FA)}

Figure $9 \mathrm{C}$ displays the group averages of the contoured area which captured $25 \%$ of gaze fixations. FA in $\mathrm{NMF}_{\mathrm{Rd}}$ was small, only $\sim 1.6 \mathrm{deg}^{2}[1.43,1.87]$, increasing for $\mathrm{N}_{\mathrm{Rd}}$ conditions to $\sim 2.7 \operatorname{deg}^{2}[2.3,3.14]$, but diminished to $1.1 \operatorname{deg}^{2}[.87,1.3]$ in $F_{R d}$ conditions. The ANOVA (see Table 2) revealed main effects of both Road and Flow, but no interaction. Contrasts showed that both $\mathrm{F}_{\mathrm{Rd}}\left(F(1,8)=50.35, p<.001, \eta_{\mathrm{p}}^{2}=.86\right)$ and $\mathrm{N}_{\mathrm{Rd}}\left(F(1,8)=33.39, p<.001, \mathrm{\eta}_{\mathrm{p}}{ }^{2}=.81\right)$ were significantly different to $\mathrm{NMF}_{\mathrm{Rd}}$. Contrasts also showed that the FA for $\mathrm{SL}_{\mathrm{F}}$ was significantly larger than $\operatorname{VE}_{F}\left(F(1,8)=11.68, p=.009, \eta_{p}^{2}=.51\right)$, whereas the $F A$ for $\mathrm{FS}_{\mathrm{F}}$ was no different from $\mathrm{VE}_{\mathrm{F}}\left(F(1,8)=.29, p=.6, \mathrm{\eta}_{\mathrm{p}}^{2}=.035\right)$. 


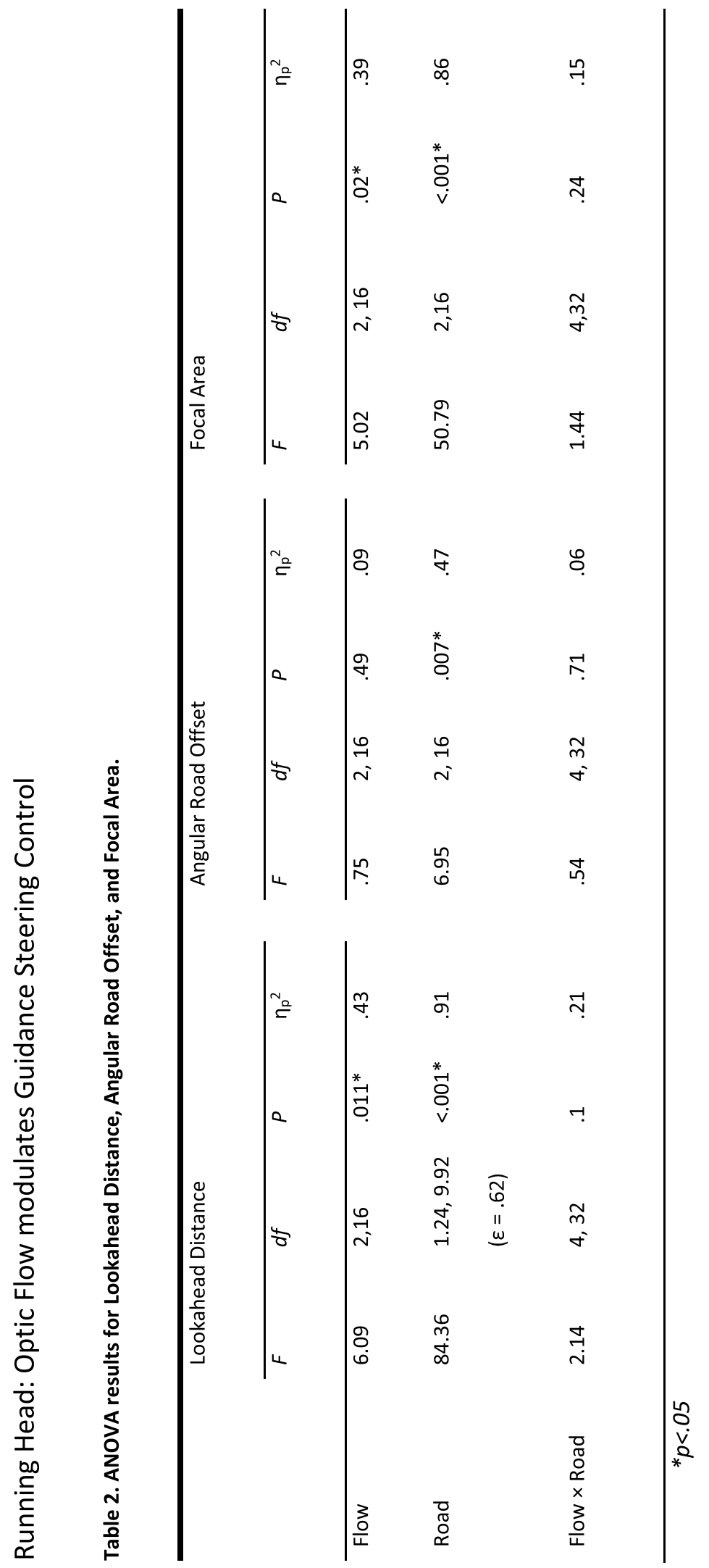


Running Head: Optic Flow modulates Guidance Steering Control

\section{Discussion}

This experiment assessed whether use of flow speed depended on road component availability, and whether eye-movements interacted with flow or road properties. Removing near or far road-edge information caused systematic changes in FISB scores, suggesting that use of flow is in part determined by the availability of road segments. The same basic pattern was observed in both Constrained and Unconstrained gaze conditions: the smallest FISB was observed in $\mathrm{N}_{\mathrm{Rd}}$ conditions with greater FISB in $\mathrm{F}_{\mathrm{Rd}}$ and $\mathrm{NMF}_{\mathrm{Rd}}$ conditions.

\section{Combining flow speed with road-edges}

Two alternative frameworks were presented in the Introduction: the Modulation Hypothesis (H1) and the Weighted Combination Hypothesis ( $\mathrm{H} 2)$. Each framework was accompanied by three competing hypotheses that predicted mutually exclusive patterns of FISB (Figures 1 \& 2). A crucial difference between $\mathrm{H} 1$ and $\mathrm{H} 2$ is whether FISB increases or decreases when the road-edge signal is made weaker by removing road-edge components. Under a weighted combination framework, a less reliable road-edge signal would be down-weighted, and flow information would be up-weighted (Ernst \& Banks, 2002; Wilkie \& Wann, 2002, 2003a). In the current experiment, there is no evidence for FISB increasing when road-edge components were selectively removed, allowing $\mathrm{H} 2$ to be rejected. Instead, FISB reduced during $\mathrm{N}_{\mathrm{Rd}}$ conditions, indicating that the utility of flow speed is boosted by the presence of road-edges, which is compatible with the framework presented under $\mathrm{H} 1$.

Across both Constrained and Unconstrained gaze conditions FISB significantly reduced for $N_{R d}$ but not for $F_{R d}$ (relative to $N_{M} F_{R d}$ ). This suggests that the contribution of flow speed to steering control is selective and depends on the nature of the control task, which is in contrast 
Running Head: Optic Flow modulates Guidance Steering Control

with previous research suggesting that a strong flow signal is ubiquitously useful for control (Chatziastros et al., 1999). This pattern also allows H1C to be rejected. Instead, the observed FISB results are most similar to the pattern predicted by H1B: whereby flow speed is predominantly useful for guidance level control.

\section{The effect of road component availability on two-level steering}

Other measures of steering behaviour (RMSE and SWJ; see Figure 7), can be used to examine further whether changes in flow-use corresponded with changes in steering behaviours. Smooth steering, but high positional error, are characteristic of guidance control; conversely, jerky steering, with low error, are characteristic of compensatory control (Land \& Horwood, 1995; Land, 1998). Steering was smoothest and most accurate when a full road was available. When near road-edge information was removed, deviation from the road centre increased and accuracy decreased. Levels of jerk and RMSE for $\mathrm{NMF}_{\mathrm{Rd}}$ and $\mathrm{F}_{\mathrm{Rd}}$ conditions were fairly consistent across gaze conditions ${ }^{6}$. This is likely due to similar gaze behaviours being employed - in Unconstrained gaze, participants looked approximately .9s ahead on the desired future path (the retinal difference between fixating at .9s and fixating at $1.2 \mathrm{~s}-$

\footnotetext{
${ }^{6}$ In Experiment 1 jerk was larger for $F_{R d}$ compared to $\mathrm{NMF}_{\mathrm{Rd}}$, which would not necessarily be predicted under the framework of two-level steering (Salvucci \& Gray, 2004). It was observed post-experiment that the method of continuously updating the far road-edges caused the update rate to reduce to $\sim 35 \mathrm{~Hz}$ in $\mathrm{F}_{\mathrm{Rd}}$ (as highlighted in the method). In Experiment 2, with a consistent update rate of $60 \mathrm{~Hz}$, $\mathrm{F}_{\mathrm{Rd}}$ jerk was equivalent to $\mathrm{NMF}_{\mathrm{Rd}}$. Therefore, it is likely that the instability effects in Experiment 1 were due to the slower refresh rate (Cloete \& Wallis, 2011) and not due to removing near road-edges.
} 
Running Head: Optic Flow modulates Guidance Steering Control

Constrained gaze - is only $1.4^{\circ}$ in these displays). Similar gaze behaviours have been observed previously when drivers have been instructed to steer a centre course (Wilkie et al., 2008).

Steering behaviour for $\mathrm{N}_{\mathrm{Rd}}$ varied across gaze conditions. During Unconstrained gaze, driving became jerkier, more errorful, and was characterised by greater understeering. Jerkier steering is a predictable consequence of removing anticipatory information, and reflects a switch to compensatory control: drivers cannot anticipate the future steering requirements, so need to operate with a large gain to keep positional error within acceptable limits (Land \& Horwood, 1995; Land, 1998). The increased error suggests that a locomotor speed of $13.41 \mathrm{~ms}$ on a $60 \mathrm{~m}$ radius $\left(12.81^{\circ} / \mathrm{s}\right)$ bend was too fast and tight a bend for compensatory control to fully 'take over' guidance control. However, when gaze was constrained, removing guidance level road-edge information caused steering behaviour to become jerkier, but positional error remained the same as when a full road was available. A critical difference between the two gaze conditions is that in unconstrained conditions participants moved their gaze closer, to around half a second ahead (rather than 1.2s). This temporal distance is similar to that previously found for compensatory control (Land, 1998); although it is often assumed that near road-edge information would naturally be sampled through peripheral vision (Salvucci \& Gray, 2004). The obvious advantage of re-orienting gaze would be to obtain task-relevant information (gauging lateral distance from road-edges). However, looking closer in the scene will reduce the guidance cues that are available, such as extra-retinal direction (Authié, Hilt, N'Guyen, Berthoz, \& Bennequin, 2015; Wilkie \& Wann, 2003a, 2005). It seems that forcing participants to look $1.2 \mathrm{~s}$ ahead on the path can actually make steering smoother $\left(\mathrm{N}_{\mathrm{Rd}}\right.$ jerk in Constrained gaze conditions is about half that of Unconstrained gaze) presumably because of the presence of two additional sources of guidance information: extra-retinal direction 
Running Head: Optic Flow modulates Guidance Steering Control

(Wilkie \& Wann, 2002), as well as target drift of the fixation cross itself (Mars, 2008). It appears that additional guidance information reduced the demands on compensatory control, so that performance was fairly accurate even when guidance level road-edge information was removed.

Successful 'switching' to compensatory control (accepting some positional error at the expense of jerk) has been observed in previous studies (Chatziastros et al., 1999; Cloete \& Wallis, 2011; Land \& Horwood, 1995), but not in others (Frissen \& Mars, 2014). Based on the current results, one explanation for the differences between 'unsuccessful' and 'successful' compensation is the degree to which gaze can be directed forwards. In Frissen \& Mars (2014) there was a large systematic increase in both jerk and positional error from a $60 \%$ upper-field mask, to an $80 \%$ upper-field mask, to a $100 \%$ upper-field mask, whereas the same increases in opacity of a lower-field mask did not impair performance markedly. Their conclusion was that the 'compensatory process is more robust to visual degradation than the anticipatory process' (Frissen \& Mars, 2014, p. 499). Our results may add additional nuance to this interpretation, because it is possible that the effects of a weaker guidance signal (due to mask degradation) were exacerbated by participants also looking less frequently into the upper mask at high degradation levels (and presumably not at all when the upper mask was 100\% degraded). Based on Experiment 1's results, a relocation of gaze to lower regions may have precipitated some of the observed decrease in both steering smoothness and steering accuracy in Frissen \& Mars (2014).

Whilst gaze behaviour during slow-speed locomotion can be captured by a trade-off between the need for direct control (near gaze) and the need for anticipation (distant gaze; Vansteenkiste, Cardon, D'Hondt, Philippaerts, \& Lenoir, 2013), the evidence presented here 
Running Head: Optic Flow modulates Guidance Steering Control

suggests that this model does not adequately generalise to high-speed driving, where the need for anticipation is great (Lehtonen, Lappi, Kotkanen, \& Summala, 2013) and near gaze can be detrimental to performance. Instead, our evidence suggests that a driver has a better chance of adapting to changes in road-edge availability if they maintain a gaze strategy of looking toward the desired future path approximately 1-2s ahead (Wilkie et al., 2008).

\section{Experiment 2: Examining the contribution of Flow Speed and Road}

Experiment 1 demonstrated that flow speed seems to be selectively useful for guidance control, rather than compensatory control, and highlighted the importance of controlling for gaze when manipulating road-edge information. However, the way in which guidance and compensatory road-edge signals are combined to provide a steering solution remains somewhat unclear. To examine the nature of the interaction between flow and guidance information the number of road-edge conditions was increased to include various combinations of Near, Middle and/or Far road-edge information. Gaze fixations were controlled through the use of 'constrained' gaze conditions throughout.

One outstanding issue is how flow speed influences guidance control. Wilkie \& Wann (2003a) showed that smooth, curved trajectories can be modelled using inputs of retinal flow that are crudely quantized on a 0-3 point scale, showing that high sensitivity to changes in perceptual input is not necessarily essential for smooth steering. It is possible that the observed FISB in Experiment 1 are very simple biases linked with a general impression of going 'faster', or 'slower', thus the steering response is only crudely adjusted. To determine whether steering responses are precisely influenced by flow speed (i.e. oversteering continues to increase as flow speed increases), or whether there is a far coarser relationship (e.g. the same amount of 
Running Head: Optic Flow modulates Guidance Steering Control

oversteering occurs whenever flow is considered 'fast') a wider range of flow speeds were generated to see whether the magnitude of flow speed is directly related to the amount of steering bias.

\section{Method}

The same apparatus as Experiment 1 was used, although participants were not eye-tracked since only constrained gaze conditions were examined (and experiment 1 confirmed compliance with these fixation requirements). All participants received the same written instructions as Experiment 1 . They then completed 20 practice trials ( 2 minutes) of veridical flow, starting with a complete road and then being exposed to each road condition for both left and right bends. For this experiment, there were five flow manipulations, labelled by their speed proportional to veridical ( $F L_{.5}, F L_{.75}, \mathrm{FL}_{1}, \mathrm{FL}_{1.25}, \mathrm{FL}_{1.5}$ ). With this notation the flow manipulations in Experiment 1 would be $\mathrm{FL}_{.66}$ and $\mathrm{FL}_{1.33}$. There were 8 different road conditions, with three, two, one or no visible road-edge components, as shown in Figure 5: $N M F_{R d}, N M_{R d}, M F_{R d}, N_{R d}, M_{R d}, F_{R d}, N F_{R d}$, and INV $V_{R d}$. The presence of the fixation cross meant we could include an extra condition, labelled the 'Invisible Road' (Figure 5). In this condition, road-edges were completely removed, so the predominant information made available to participants was the flow field and the fixation point. This condition was included to provide an estimate of the maximum effect of flow speed since no road-edge information was available to reduce steering errors, but the fixation cross still provided a guidance signal. Note that participants always experienced the full road viewing condition before their first 'invisible' road condition to ensure they steered (as much as possible) as if they were on a bending road. There were six trials in each condition, each six seconds long, resulting in an 
Running Head: Optic Flow modulates Guidance Steering Control

experiment running time of 24 minutes. A brief rest break was inserted at the half-way point to alleviate fatigue.

\section{Participants}

20 participants (10 females, 10 males, ages 20 to 35, mean 25.7 years), all having normal or corrected-to-normal vision, took part in the experiment. All participants were naïve as to the purpose of the study and had not participated in Experiment 1. Eighteen participants held driving licenses (average time since driving test $=6.6$ years), and the remaining two had some limited driving experience (go-karting and driving lessons). All participants gave informed consent and the study was approved by the University of Leeds Ethical Committee (Ref: 140225), and complied with all guidelines as set out in the declaration of Helsinki.

\section{Analysis}

The same steering measures as Experiment 1 were used: SB, RMSE and SWJ. Experiment 2 had an expanded experimental design so that improved FISB estimates could be calculated to measure overall reliance upon flow under each road-edge condition. FISB scores were calculated for each participant by finding the slope of the linear regression fitted to the steering bias estimates across the five flow speeds for each road condition. The slopes were fitted by logarithmically transforming the data then performing weighted linear regression (see Appendix A for a detailed explanation). 
Running Head: Optic Flow modulates Guidance Steering Control

\section{Results}

A 5 (Flow) $\times 8$ (Road) repeated measures ANOVA was conducted on steering measures (FISB, Steering Bias, Steering Error, and Steering Smoothness). Planned simple contrasts compared every level to the control condition $\left(\mathrm{NMF}_{\mathrm{Rd}}+\mathrm{FL}_{1}\right)$.

\section{Flow-Induced Steering Bias (FISB)}

FISB measures the extent to which different flow speeds influenced steering under each roadedge condition. A large positive FISB indicates relative understeering in slower flow conditions and/or oversteering in faster flow conditions, whereas smaller FISB indicates little sensitivity to flow speed changes exhibited in steering bias (Figure 10). Consistent with Experiment 1 the largest FISB was seen when guidance information was present $\left(\mathrm{NMF}_{\mathrm{Rd}}, \mathrm{F}_{\mathrm{Rd}}\right.$ and $I N V_{\mathrm{Rd}}$ conditions; Figure 10). Compared to $\mathrm{NMF}_{\mathrm{Rd}}$, FISB was reduced to approximately $80 \%$ during $\mathrm{MF}_{\mathrm{Rd}}$, and reduced to $55 \%$ during $\mathrm{NM}_{\mathrm{Rd}}, \mathrm{M}_{\mathrm{Rd}}$, and $\mathrm{NF}_{\mathrm{Rd}}$ conditions. When guidance information was weakest ( $\left.N_{R d}\right)$ the smallest FISB was observed, which was $\sim 42 \%$ of that during $\mathrm{NMF}_{\mathrm{Rd}}$ conditions (for convenience those with similar FISB scores have been grouped in Figure 10 , these groups will be considered further in the qualitative analysis of trajectories). A oneway repeated measures ANOVA was conducted (with Greenhouse-Geisser correction applied; $\varepsilon=.58)$ demonstrating a significant effect of road condition $(F(4.02,76.45)=10.53, p<.001$, $\eta_{p}{ }^{2}=.35$ ). Simple contrasts comparing every level to $\mathrm{NMF}_{\mathrm{Rd}}$ (Table 3 ) revealed that $\mathrm{F}_{\mathrm{Rd}}$ and INV $V_{R d}$ were the only conditions not significantly different to $N_{M} F_{R d}$, with effect sizes increasing as guidance information from Far and Middle road-edge information was removed $\left(\mathrm{MF}_{\mathrm{Rd}}<\right.$ $N F_{R d}<N M_{R d}<M_{R d}<N_{R d}$. The only exception to this pattern is the FISB for $N F_{R d}$ which was significantly smaller than $\mathrm{NMF}_{\mathrm{Rd}}$. Given that similar guidance and compensatory signals should be available in both conditions it seems that the 'disconnected' nature of the road- 
Running Head: Optic Flow modulates Guidance Steering Control

edge components in $\mathrm{NF}_{\mathrm{Rd}}$ is sufficient to disrupt and reduce guidance control (and thereby reduce FISB). This finding contrasts with the Land \& Horwood suggestion of an optimal region midway between far and near regions, as well as Salvucci \& Gray's (2004) stipulation that steering can be accurate as long as both a guidance and compensatory signal are available.

Table 3. Contrasts for FISB in Experiment 2

\begin{tabular}{lllll}
\hline $\begin{array}{l}\text { Contrast (vs } \\
N M F)\end{array}$ & $D F$ & $F$ & $P$ & $\eta_{p}^{2}$ \\
\hline $\mathrm{N}_{\mathrm{Rd}}$ & 1,19 & 53.96 & $<.001$ & .74 \\
$\mathrm{NM}_{\mathrm{Rd}}$ & 1,19 & 28.45 & $<.001$ & .6 \\
$\mathrm{NF}_{\mathrm{Rd}}$ & 1,19 & 21.01 & $<.001$ & .53 \\
$\mathrm{M}_{\mathrm{Rd}}$ & 1,19 & 31.99 & $<.001$ & .63 \\
$\mathrm{MF}_{\mathrm{Rd}}$ & 1,19 & 4.42 & .049 & .19 \\
$\mathrm{~F}_{\mathrm{Rd}}$ & 1,19 & .19 & .66 & .01 \\
$\mathrm{INV}_{\mathrm{Rd}}$ & 1,19 & .17 & .68 & .01 \\
\hline
\end{tabular}

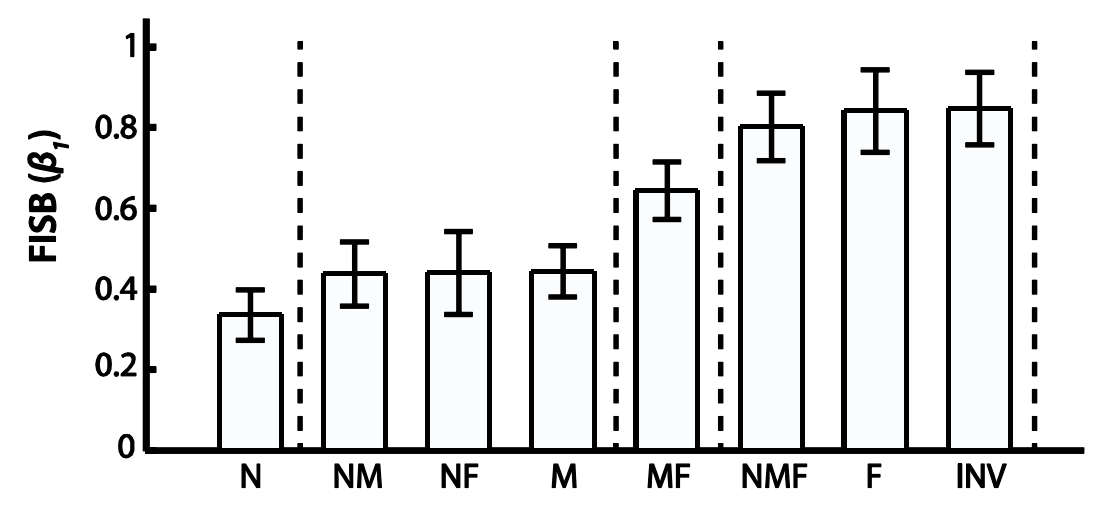

Figure 10 FISB scores for Experiment 2 (which are $\beta_{1}$ regression values obtained from weighted linear regression; see Appendix A). The dotted lines represent the 'groupings' identified within the text. Road conditions are ordered by FISB magnitude. Error bars represent standard error of the mean.

\section{Steering Bias (SB)}

Figure 11A shows SB for every flow level, across all road-edge conditions. As per Experiment

1 there was a general trend to oversteering when flow speed was increased, and 
Running Head: Optic Flow modulates Guidance Steering Control

understeering when flow speed was decreased, but this effect varied according to which road segments were visible. The results of the ANOVA are in Table 4. There were significant main effects of both Flow and Road, and also a Road $\times$ Flow interaction. Planned contrasts revealed that the interaction was caused by the magnitude of steering response induced by manipulating flow speed reducing in some road conditions compared to $\mathrm{NMF}_{\mathrm{Rd}}$. In particular, the understeering-inducing effects of $F L .5$ was significantly reduced in $N_{R d}(F(1,19)=13.43, p$ $\left.=.002, \eta_{p}^{2}=.41\right), \mathrm{NM}_{\mathrm{Rd}}\left(\mathrm{F}(1,19)=18.81, \mathrm{p}<.001, \mathrm{\eta}_{\mathrm{p}}{ }^{2}=.5\right), \mathrm{M}_{\mathrm{Rd}}(\mathrm{F}(1,19)=31.28, \mathrm{p}<.001$, $\left.\eta_{p}{ }^{2}=.62\right)$, and $N F_{R d}\left(F(1,19)=6.05, p=.024, \eta_{p}^{2}=.24\right)$.
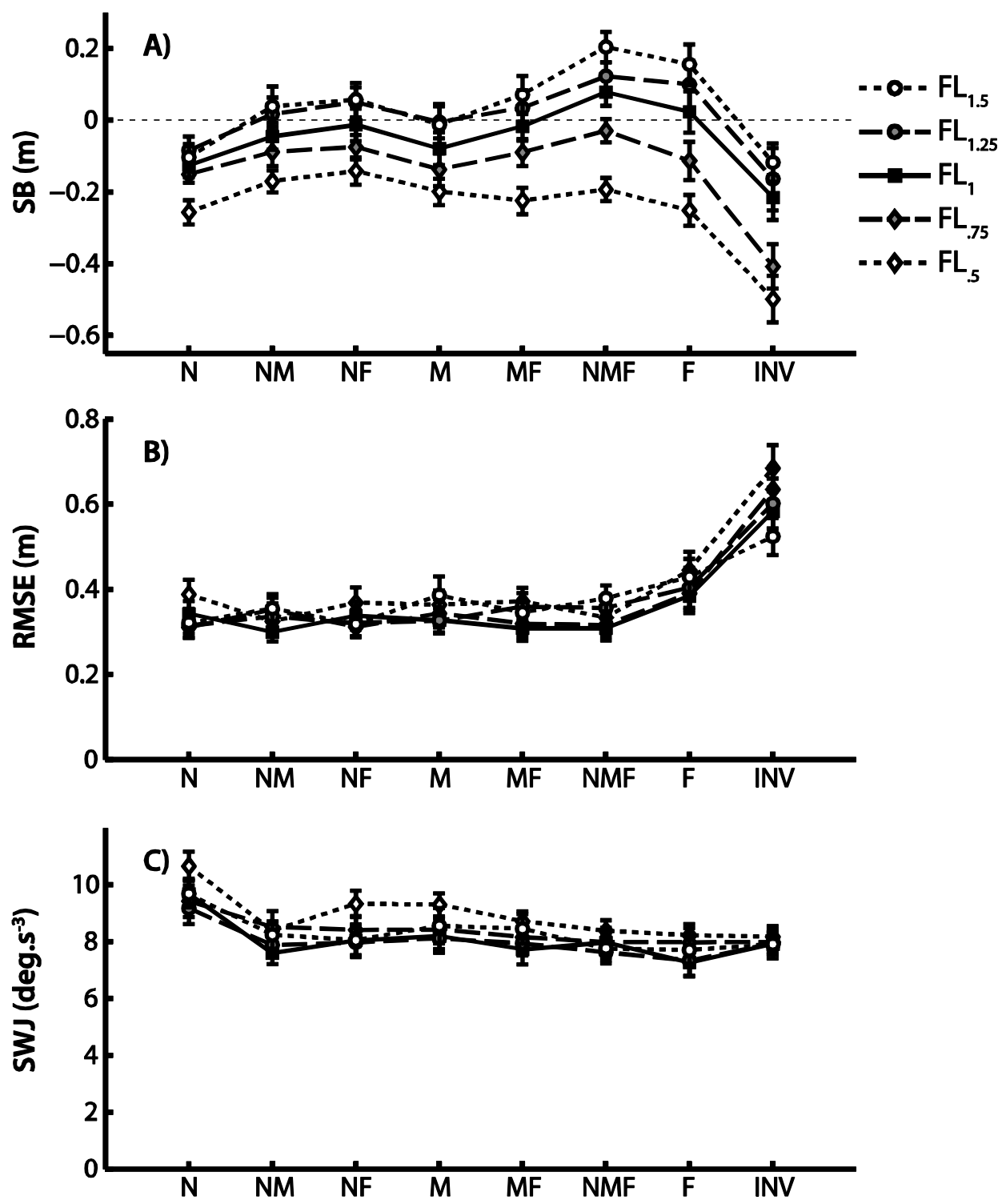

Road Condition 
Running Head: Optic Flow modulates Guidance Steering Control

Figure 11. All steering performance measures across $\mathrm{FL}_{1.5}$ (empty circles, dotted line), $\mathrm{FL}_{1.25}$ (grey circles, dashed line), $\mathrm{FL}_{1}$ (sold squares, solid line), FL.75 (grey diamonds, dashed line), and FL.5 (empty diamond, dotted line), showing, B) Steering Bias, C) Steering Error, and D) Steering Smoothness. To facilitate comparison with Figure 10 road conditions are similarly ordered by FISB magnitude. Error bars represent standard error of the mean.

\section{Steering Error (RMSE)}

RMSE gives an indication of total deviation from the road centre (Figure 11B). RMSE is fairly constant across road conditions ( $.35 \mathrm{~m})$, with the main exception being the increase in error for $F_{R d}(M=.41 m,[.29, .41], S E M=.04)$ and for $\operatorname{INV}_{R d}(M=.61 \mathrm{~m},[.51, .7]$, SEM $=.05)$. The results from the ANOVA are shown in Table 4. There is no effect of Flow, but there is a significant main effect of Road and a significant Flow $\times$ Road interaction. The main effect is driven by the RMSE score increasing, compared to $\mathrm{NMF}_{R d}$, for $F_{R d}(F(1,19)=13.34, p=.002$, $\left.\eta_{p}^{2}=.41\right)$ and $\operatorname{INV}_{R d}\left(F(1,19)=13.34, p<.001, \eta_{p}^{2}=.75\right)$. There does not appear to be a systematic pattern causing the interaction.

\section{Steering Smoothness (SWJ)}

Figure $11 \mathrm{C}$ shows SWJ, which approximates to rapid steering corrections. The ANOVA (Table 4) revealed significant main effects of Flow and Road, but no interaction. The main effect of Flow was due to $F L .5\left(F(1,19)=30.08, p<.001, \eta_{p}{ }^{2}=.61\right)$ and $F L .75(F(1,19)=13.22, p=.002$, $\eta_{p}{ }^{2}=.41$ ) both causing significantly jerkier behaviour than $\mathrm{FL}_{1}$. The main effect of Road was caused by significantly jerkier behaviour, compared with $\mathrm{NMF}_{\mathrm{Rd}}$, during $\mathrm{N}_{\mathrm{Rd}}(\mathrm{F}(1,19)=57.89$, $\left.p<.001, \eta_{p}^{2}=.75\right), M_{R d}\left(F(1,19)=22.61, p<.001, \eta_{p}^{2}=.54\right), M F_{R d}(F(1,19)=5.46, p=.031$, $\left.\eta_{p}{ }^{2}=.22\right)$, and $N F_{R d}\left(F(1,19)=9.16, p=.007, \eta_{p}^{2}=.33\right)$. The jerkier steering in $\mathrm{NF}_{R d}($ compared to $\mathrm{NMF}_{\mathrm{Rd}}$ ) is consistent with the conclusion that separating the road into two disconnected 
Running Head: Optic Flow modulates Guidance Steering Control

segments caused participants to shift away from smooth guidance control towards a compensatory steering mode. 


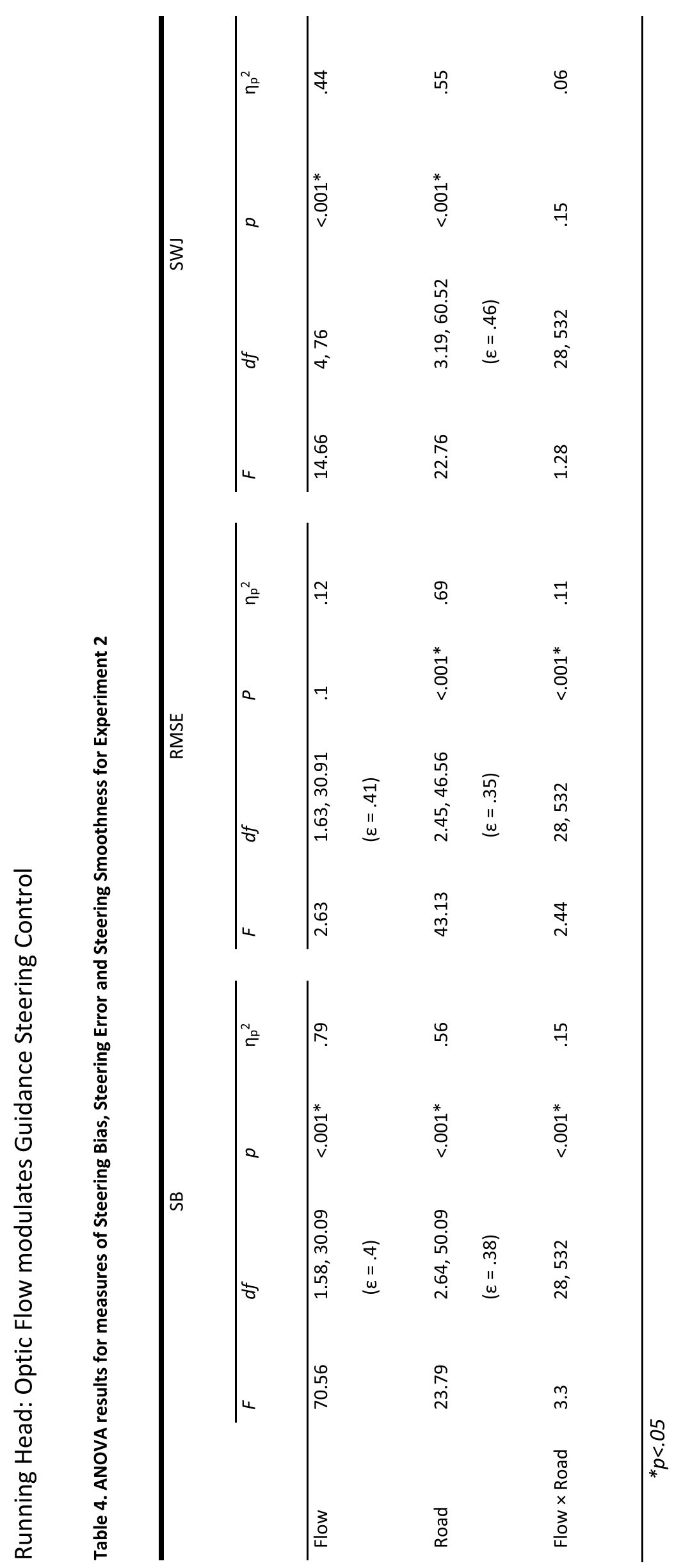


Running Head: Optic Flow modulates Guidance Steering Control

\section{Analysing Trajectory Development}

To examine in more detail how steering changed across time we calculated the average trajectory bias for each frame (across all participants). The calculation of error bounds (shaded regions in Figure 12) demonstrates where in the trajectory participants' behaviour was more or less consistent. It can be seen that $\mathrm{NMF}_{\mathrm{Rd}}$ and $\mathrm{F}_{\mathrm{Rd}}$ (Figure $12 \mathrm{~F} \& \mathrm{G}$ ) have qualitatively similar trajectories, but responses to $F_{R d}$ are more variable. This seems to be reflected in similar bias and jerk scores, but higher total deviation from the road centre for $\mathrm{F}_{\mathrm{Rd}}$ (Figure 11). Importantly, the similarity demonstrates that the equivalent FISB (Figure 10) captures qualitative similarities between the $F_{R d}$ and $N_{M M}$. On the other hand, while $I N V_{R d}$ (Figure $12 \mathrm{H}$ ) has a similar FISB, the trajectory plots show that steering behaviours during INV $V_{\mathrm{Rd}}$ are qualitatively different to when $F_{R d}$ is available. This is understandable given the weak guidance information (and lack of any road-edge information).

$N_{R d}$ has a unique profile (Figure 12A). Initially understeering develops rapidly, (akin to INV $V_{R d}$ Figure $12 \mathrm{H}$ ), however, unlike $\mathrm{INV}_{\mathrm{Rd}}$ the error is sharply corrected and the trajectory brought closer to the road centre, resulting in jerky steering with low RMSE (Figure 11B \& C). There is extensive overlap between flow levels $\mathrm{FL}_{.75}, \mathrm{FL}_{1}, \mathrm{FL}_{1.25}$, and $\mathrm{FL}_{1.5}$, which explains the low $\mathrm{FISB}$ observed in Figure 10.

There were three conditions that had very similar FISB scores: $\mathrm{NM}_{\mathrm{Rd}}, \mathrm{NF}_{\mathrm{Rd}}$, and $\mathrm{M}_{\mathrm{Rd}}$ (Figure 10), which are reflected in almost identical trajectory plots (Figure 12B-D). The trajectories are characterised by an absence of oversteering induced by increased flow speed (as seen in $\mathrm{NMF}_{\mathrm{Rd}}$ and $\mathrm{F}_{\mathrm{Rd}}$ ), and diminished understeering induced by decreasing flow speed. These similarities are also reflected in comparable SB, RMSE, and SWJ between these conditions (Figure 11). 
Running Head: Optic Flow modulates Guidance Steering Control

$M_{\text {Rd }}$ represents a 'transition' between the high-FISB and low-FISB conditions: it has lower FISB than $\mathrm{NMF}_{\mathrm{Rd}}$ and $\mathrm{F}_{\mathrm{Rd}}$, but higher FISB than $\mathrm{NM}_{\mathrm{Rd}}, \mathrm{NF}_{\mathrm{Rd}}$, and $\mathrm{M}_{\mathrm{Rd}}$ (Figure 10). The trajectory plot reveals a steering response that is qualitatively similar to $\mathrm{NMF}_{\mathrm{Rd}}$ and $\mathrm{F}_{\mathrm{Rd}}$ for slower flow conditions, but without the oversteering observed in $\mathrm{NMF}_{\mathrm{Rd}}$ and $\mathrm{F}_{\mathrm{Rd}}$ when flow speed is increased (compare Figure 12E to Figure 12F).

Overall Figure 12 demonstrates that the FISB groupings identified in Figure 10 also have qualitative similarities. The presence of the far road component causes more distinct trajectories across flow levels $\left(\mathrm{NMF}_{\mathrm{Rd}}, \mathrm{F}_{\mathrm{Rd}}\right.$ and $\left.\mathrm{MF}_{\mathrm{Rd}}\right)$ than when $\mathrm{F}_{\mathrm{Rd}}$ road is removed $\left(\mathrm{N}_{\mathrm{Rd}}\right.$, $N M_{R d}$, and $M_{R d}$ ). Critically, trajectories for each flow level overlap most for $N_{R d}$, when all guidance road-edge information (from the mid and far road) is removed. The main exception to this pattern is $N F_{R d}$, which is remarkably similar to $M_{R d}$ despite displaying both near and far components (therefore according to the two-level model proposed by Salvucci \& Gray, 2004, it would have been expected for $\mathrm{NF}_{\mathrm{Rd}}$ to be most similar to $\mathrm{NMF}_{\mathrm{Rd}}$ ). 


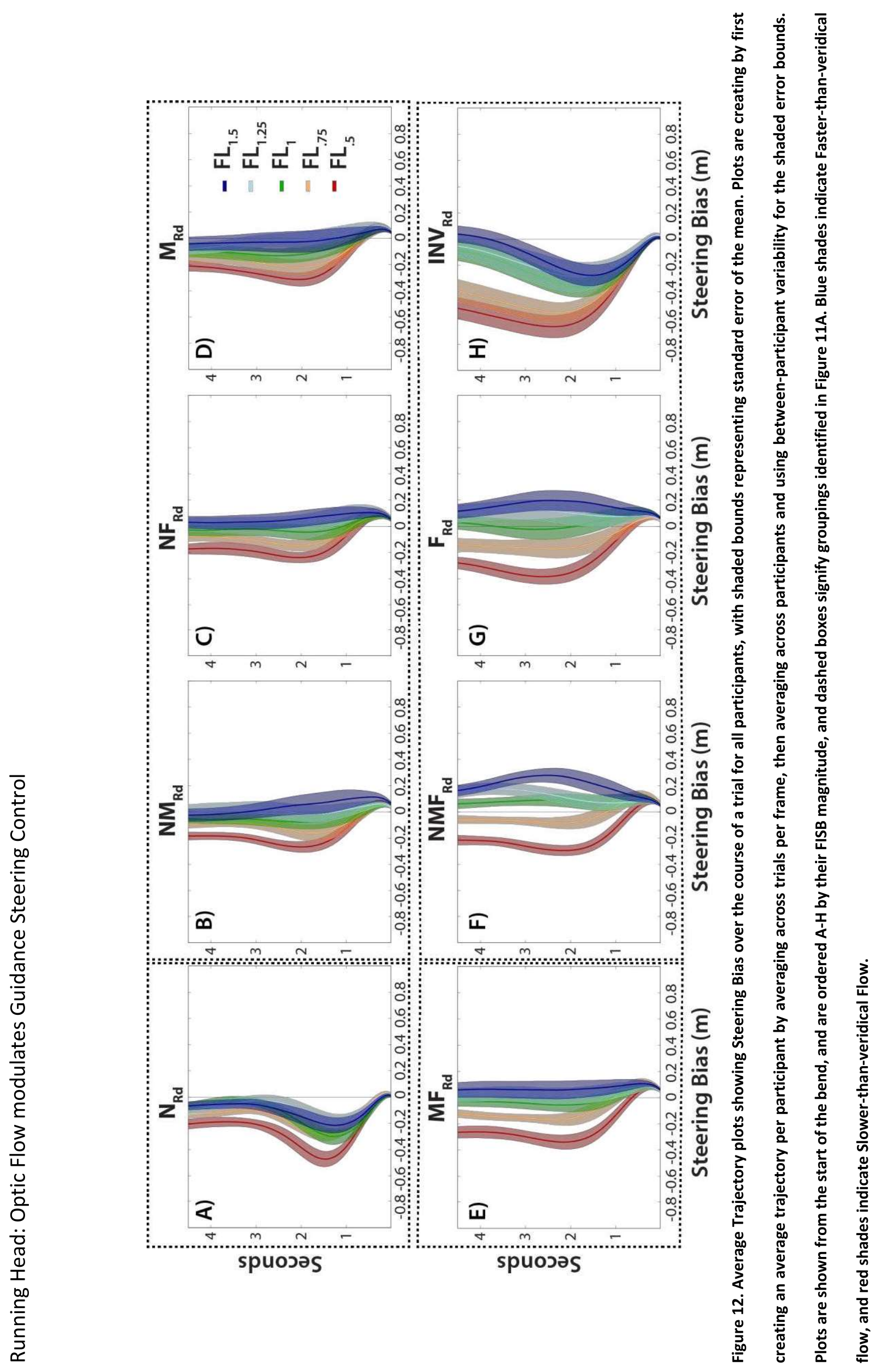


Running Head: Optic Flow modulates Guidance Steering Control

\section{Discussion}

Experiment 2 systematically varied the amount of guidance and compensatory road-edge information to test the hypothesis that flow speed modulates a guidance level control signal (Figure 1B). The overall pattern of results suggest that the presence of guidance level information is associated with an increased influence of flow speed: FISB was least for conditions where immediate position-in-lane information was available (from the Near road), but cues about the upcoming curvature requirements (from the Mid and Far segments) were removed. Combining the Near and Mid road segments (which provides some guidance information) increased FISB, and then adding the Far road segment ( $\mathrm{NMF}_{\mathrm{Rd}}$ ) increased FISB further. The key determinant of the extent to which flow influenced steering appears to be the availability and quality of guidance level information. Removing all compensatory information resulted in a FISB not markedly different from $\mathrm{NMF}_{\mathrm{Rd}}$ (and removing the Near road from $\mathrm{NMF}_{\mathrm{Rd}}$ caused only a small reduction in FISB). This pattern supports the hypothesis that the influence of flow speed is dependent upon a guidance level road-edge signal (Figure 1B).

The single exception to this general pattern is $\mathrm{NF}_{\mathrm{Rd}}$. Under an additive two-level model (e.g. Salvucci \& Gray, 2004) displaying both Near and Far components should produce similar steering behaviour to the full road $\left(\mathrm{NMF}_{\mathrm{Rd}}\right)$. In the current experiment, however, levels of jerk (Figure $11 \mathrm{C}$ ) for $\mathrm{NF}_{\mathrm{Rd}}$ are most similar to $\mathrm{M}_{\mathrm{Rd}}$, and the trajectory plots for these conditions are qualitatively very closely matched (Figure $12 C \& D$ ). These measures suggest that $\mathrm{NF}_{\mathrm{Rd}}$, which under an additive framework should produce both strong compensatory and guidance level signals, instead produced control behaviours similar to a single weakly informative signal $\left(\mathrm{M}_{\mathrm{Rd}}\right)$. It seems, then, that a complete contiguous road was treated differently compared to 
Running Head: Optic Flow modulates Guidance Steering Control

when the road boundaries were broken. This finding disagrees with a simple additive combination of the two-levels as previously proposed (Land \& Horwood, 1995; Saleh et al., 2011; Salvucci \& Gray, 2004).

Experiment 2 explored the combination of guidance and compensatory road-edge signals when gaze direction was controlled (this appears to be the first attempt to do this, see the limitations of Chatziastros et al., 1999, Cloete \& Wallis, 2011, and Land \& Horwood, 1995 as outlined earlier). A shift to compensatory control is generally identified by reduced smoothness (and smaller position errors), whereas a shift to guidance control is generally linked with greater position errors but smoother steering (Land, 1998). In the present results, there was generally little change in steering error (RMSE) and smoothness (SWJ) across road presentation conditions, with the only marked increases being observed in the 'extreme' conditions when all guidance level information from the road was removed $\left(\mathrm{N}_{\mathrm{Rd}}\right.$; low smoothness), or all compensatory level information was removed ( $\mathrm{F}_{\mathrm{Rd}}$; high steering errors). This finding supports suggestions that the visual-motor system is robust and copes fairly well with weakly informative guidance and compensatory road-edge signals (Land \& Horwood, 1995; Neumann \& Deml, 2011), however it does contradict previous research that has reported changes in instability and positional deviation measures yoked to the availability of compensatory and guidance-level road-edge information (Chatziastros, Wallis, \& Bülthoff, 1999; Land \& Horwood, 1995). However the current results do support Cloete \& Wallis (2011), who failed to find clear indicators of a shift in control strategies in response to changes in road-edge information. 
Running Head: Optic Flow modulates Guidance Steering Control

\section{General Discussion}

These experiments have confirmed that road-edge information, optic flow information and gaze direction all contribute to successful control of steering (as per Robertshaw \& Wilkie, 2008; Kountouriotis et al., 2013). Experiment 1 highlighted that steering performance was 'best' (smallest steering error, least steering bias and smoothest steering) when the full road was visible and the flow speed was veridical, but that systematically varying flow speed caused reciprocal changes in steering despite complete road-edge information. Both Experiment 1 and 2 confirmed that flow speed interacts selectively with guidance information: when only Near road-edge information was available flow speed had very little influence over steering, whereas the presence of the Far road component was usually sufficient to cause steering to exhibit flow induced steering biases (the only exception to this pattern was the Near-Far condition which appears to be treated similar to Mid road trials).

The impact of gaze behaviours was revealed in Experiment 1. Unconstrained gaze conditions highlighted that participants tended to look in the same distal region across road-edge conditions, except when only Near road-edge information was present. Shifting gaze down to look between the near road-edges was the natural response in this case, and this shift was accompanied by reduced influence of flow speed over steering (compared to when gaze was constrained and directed towards a distal fixation point). It seems that even when there is no road-edge information available, the guidance information supplied from constraining gaze to a far point on the future path was still sufficient to cause flow speed to have some influence over steering (though nowhere near as large an effect as when far road-edge information was visible). It follows that differences observed between the constrained gaze conditions in these experiments and the results reported in previous studies with unconstrained gaze (e.g. Chatziastros, Wallis, \& Bülthoff, 1999; Frissen \& Mars, 2014; Land \& Horwood, 1995) could be 
Running Head: Optic Flow modulates Guidance Steering Control

explained by free gaze fixation of the near road when far road-edges are removed. This is an important issue for future two-level steering research: a full description of human steering control solutions will not be achieved unless the interaction between gaze behaviours, retinal flow and guidance and compensatory road-edge signals are fully understood.

Whilst the experimental findings are most consistent with the Hypothesis $1 \mathrm{~B}$ (outlined in Figure 1B), it should be emphasised that rather than the 'all or nothing' hypotheses outlined here, the pattern of results across both experiments are consistent with guidance information having a variable weighting depending upon the strength of the signal (Frissen \& Mars, 2014). It can be seen that guidance information gradually increases across the following conditions: no guidance ( $N_{R d}$, unconstrained gaze), guidance from gaze $\left(N_{R d}\right)$, guidance from gaze and limited guidance from the Mid road component $\left(\mathrm{NM}_{\mathrm{Rd}}\right)$, full guidance $\left(\mathrm{NMF}_{\mathrm{Rd}}\right)$. The steering behaviours reflect this gradual increase, with the influence of flow speed mapping closely to the strength of the guidance signal in each condition.

There are currently no steering models that fully capture the steering behaviours observed in these experiments. Flow-inspired steering models (e.g. Fajen \& Warren, 2003; Wilkie \& Wann, 2003a; Wann \& Swapp, 2000) have developed relatively independently of two-level steering models (Mars et al., 2011; Salvucci \& Gray, 2004). This present work attempted to reconcile the two approaches by assessing whether flow speed information can be understood within a two-level framework (Donges, 1978). The results demonstrate that not only does flow speed influence steering in the presence of road-edge information, but that this use is predominantly through an interaction with guidance-level control.

One question raised by these experiments is how does flow speed become incorporated into steering control? Flow speed by itself does not provide sufficient directional information for 
Running Head: Optic Flow modulates Guidance Steering Control

effective steering, and so it seems unlikely that it is used directly to steer. Flow speed does, however, provide speed information that could be useful for estimating the likely position of some future goal, and could simply scale the current steering so that a faster rate of travel leads to a greater steering responses based on the directional inputs from far road-edge information. It should be emphasised, however, that the results are not consistent with simply faster reaction times for faster moving flow: Figure 12 shows that the timing of steering responses were unaffected by flow speed (all trajectories were initiated at an equivalent time point). Rather flow speed appears to modulate steering responses in a much more nuanced fashion. It is possible that such behaviours could be elicited by a modified version of the Salvucci \& Gray (2004) model whereby the influence of the far point is varied according to flow speed, however, such an implementation would not account for the steering behaviours observed when separate near and far road-edges were present (where we actually observe similar steering to when a single Mid component was present), nor would it account for the FISB observed in conditions without far road-edge information.

It seems that whilst our results are most consistent with flow speed modulating guidance level steering control (Figure 1B), such a mechanism is not simple to implement via a standard feedback modelling solution (such as using visual angle to a point to control steering e.g. Salvucci \& Gray, 2004). It is unclear, at this stage, whether flow speed modulates guidancelevel information as part of a feedback loop, or whether it acts concurrently under traditional 'open-loop' control (Donges, 1978). There have, however, been many steering models that incorporate directional information from flow (rather than speed), in a closed-loop manner (Fajen \& Warren, 2003; Warren et al., 2001; Wilkie \& Wann, 2002). What is clear is that future work will need to determine how other sources of information from retinal flow (e.g. flow 
Running Head: Optic Flow modulates Guidance Steering Control

direction) are sampled via active gaze and combined with compensatory and guidance information from road-edges to support successful steering control. In order to translate such laboratory findings to real-world driving it will also be important to ensure that these models apply to drivers steering their preferred course along bends of varying curvature, whilst being free to look where they like in the scene.

\section{Acknowledgements}

CDM was funded by the Emma \& Leslie Reid Foundation. The authors would like to thank Otto Lappi and Franck Mars for insightful comments on early versions of the manuscript. Thanks also to John Wann for the loan of the eye-tracker used in this experiment. 
Running Head: Optic Flow modulates Guidance Steering Control

\section{References}

Authié, C. N., Hilt, P. M., N'Guyen, S., Berthoz, A., \& Bennequin, D. (2015). Differences in gaze anticipation for locomotion with and without vision. Frontiers in Human Neuroscience, 9(312), 1-16.

Authié, C. N., \& Mestre, D. R. (2012). Path curvature discrimination: dependence on gaze direction and optical flow speed. PloS One, 7(2), e31479.

Beall, A. C., \& Loomis, J. M. (1996). Visual control of steering without course information. Perception, 25(4), 481-94.

Billington, J., Field, D. T., Wilkie, R. M., \& Wann, J. P. (2010). An fMRI study of parietal cortex involvement in the visual guidance of locomotion. Journal of Experimental Psychology. Human Perception and Performance, 36(6), 1495-507.

Chattington, M., Wilson, M., Ashford, D., \& Marple-Horvat, D. E. (2007). Eye-steering coordination in natural driving. Experimental Brain Research, 180, 1-14.

Chatziastros, A., Wallis, G. M., \& Bülthoff, H. H. (1999). The Effect of Field of View and Surface Texture on Driver Steering Performance. In A. G. Gales, I. D. Brown, C. M. Haslegrave, \& S. P. Taylor (Eds.), Vision in Vehicles VII (pp. 253-260). Amsterdam: Elsevier.

Cheng, J., \& Li, L. (2011). Perceiving path from optic flow. Journal of Vision, 11(1):22, 1-15.

Cloete, S. R., \& Wallis, G. M. (2009). Limitations of feedforward control in multiple-phase steering movements. Experimental Brain Research, 195(3), 481-7.

Cloete, S. R., \& Wallis, G. M. (2011). Visuomotor control of steering: the artefact of the matter. Experimental Brain Research, 208(4), 475-89.

Desmurget, M., \& Grafton, S. (2000). Forward modeling allows feedback control for fast reaching movements. Trends in Cognitive Sciences, 4(11), 423-431.

Donges, E. (1978). A two-level model of driver steering behavior. Human Factors, 20(6), 691707.

Ernst, M. O., \& Banks, M. S. (2002). Humans integrate visual and haptic information in a statistically optimal fashion. Nature, 415(6870), 429-33.

Faisal, a A., Selen, L. P. J., \& Wolpert, D. M. (2008). Noise in the nervous system. Nature Reviews. Neuroscience, 9(4), 292-303.

Fajen, B. R., \& Warren, W. H. (2003). Behavioral dynamics of steering, obstacle avoidance, and route selection. Journal of Experimental Psychology. Human Perception and Performance, 29(2), 343-362.

Frissen, I., \& Mars, F. (2014). The effect of visual degradation on anticipatory and compensatory steering control. Quarterly Journal of Experimental Psychology, 67(3), 499-507.

Godthelp, H. (1986). Vehicle control during curve driving. Human Factors: The Journal of the Human Factors and Ergonomics Society, 28(2), 211-221. 
Itkonen, T., Pekkanen, J., \& Lappi, O. (2015). Driver Gaze Behavior Is Different in Normal Curve Driving and when Looking at the Tangent Point. Plos One, 10(8), e0135505.

ISO 8855: 2011. Road Vehicles - vehicle dynamics and road-holding ability - vocabulary. Geneva: International Organisation for Standardization, 2011.

Jagacinski, R. J., \& Flach, J. M. (2003). Control Theory for Humans: Quantitative Approaches to Modeling Performance. Mahwah, NJ: Erlbaum.

Kandil, F. I., Rotter, A., \& Lappe, M. (2009). Driving is smoother and more stable when using the tangent point. Journal of Vision, 9, 1-11.

Kandil, F. I., Rotter, A., \& Lappe, M. (2010). Car drivers attend to different gaze targets when negotiating closed vs. open bends. Journal of Vision, 10(4):24, 1-11.

Kim, N. G., \& Turvey, M. T. (1998). Visually perceiving heading on circular and elliptical paths. Journal of Experimental Psychology. Human Perception and Performance, 24(6), 1690704.

Kim, N. G., \& Turvey, M. T. (1999). Eye movements and a rule for perceiving direction of heading. Ecological Psychology, 11, 233-248.

Kountouriotis, G. K., Floyd, R. C., Gardner, P. H., Merat, N., \& Wilkie, R. M. (2012). The role of gaze and road edge information during high-speed locomotion. Journal of Experimental Psychology: Human Perception and Performance, 38 (3), 687-702

Kountouriotis, G. K., Mole, C. D., Merat, N., \& Wilkie, R. M. (2016). The need for speed: global optic flow speed influences steering. Royal Society Open Science, $3: 160096$

Kountouriotis, G. K., Shire, K. A., Mole, C. D., Gardner, P. H., Merat, N., \& Wilkie, R. M. (2013). Optic flow asymmetries bias high-speed steering along roads. Journal of Vision, 13(10), $1-9$.

Kountouriotis, G. K., \& Wilkie, R. M. (2013). Displaying optic flow to simulate locomotion: Comparing heading and steering. i-Perception, 4(5), 333-346.

Land, M. F. (1998). The visual control of steering. In L. R. Harris \& H. Jenkins (Eds.), Vision and Action (pp. 421-441). Cambridge: Cambridge University Press.

Land, M. F., \& Horwood, J. (1995). Which parts of the road guide steering? Nature, 377(6547), 339-40.

Land, M. F., \& Lee, D. N. (1994). Where we look when we steer. Nature, 369, 742-744.

Lappi, O. (2014). Future path and tangent point models in the visual control of locomotion in curve driving. Journal of Vision, 14(12):21, 1-22.

Lappi, O., \& Pekkanen, J. (2013). Beyond the tangent point: Gaze targets in naturalistic driving. Journal of Vision, 13(13):11, 1-18.

Lappi, O., Pekkanen, J., \& Itkonen, T. H. (2013). Pursuit eye-movements in curve driving differentiate between future path and tangent point models. PloS One, 8(7), e68326.

Lehtonen, E., Lappi, O., Kotkanen, H., \& Summala, H. (2013). Look-ahead fixations in curve driving. Ergonomics, 56(1), 34-44.

Li, L., \& Chen, J. (2010). Relative contributions of optic flow, bearing, and splay angle 
Running Head: Optic Flow modulates Guidance Steering Control

information to lane keeping. Journal of Vision, 10, 1-14.

Li, L., Chen, J., \& Peng, X. (2009). Influence of visual path information on human heading perception during rotation. Journal of Vision, 9(3):29, 1-14.

Li, L., Sweet, B. T., \& Stone, L. S. (2006). Humans can perceive heading without visual path information. Journal of Vision, 6(9), 874-81.

Macuga, K. L., Beall, A. C., Kelly, J. W., Smith, R. S., \& Loomis, J. M. (2007). Changing lanes: Inertial cues and explicit path information facilitate steering performance when visual feedback is removed. Experimental Brain Research, 178(2), 141-150. $\mathrm{h}$

Markkula, G., Benderius, O., \& Wahde, M. (2014). Comparing and validating models of driver steering behaviour in collision avoidance and vehicle stabilisation. Vehicle System Dynamics: International Journal of Vehicle Mechanics and Mobility, 52(12), 1658-1680.

Mars, F. (2008). Driving around bends with manipulated eye-steering coordination. Journal of Vision, 8, 1-11.

Mars, F., Saleh, L., Chevrel, P., Claveau, F., \& Lafay, J.F. (2011). Modeling the visual and motor control of steering with an eye to shared-control automation. Proceedings of the Human Factors and Ergonomics Society 55th Annual Meeting - 2011, pp. 1422-1426.

Mars, F. \& Navarro, J. (2012). Where we look when we drive with or without active steering wheel control. PLOS ONE, 7(8): e43858.

Neumann, H., \& Deml, B. (2011). The two-point visual control model of steering-new empirical evidence. In V. G. Duffy (Ed.), Digital Human Modeling (pp. 493-502). Berlin: Springer.

Plöchl, M., \& Edelmann, J. (2007). Driver models in automobile dynamics application. Vehicle System Dynamics, 45 (7-8), 699-741.

Raviv, D., \& Herman, M. (1991). A new approach to vision and control for road following. Proceedings of the IEEE Workshop on Visual Motion.

Raw, R. K., Kountouriotis, G. K., Mon-Williams, M. \& Wilkie, R. M. (2012). Age differences in motor-control: Does old age mean middle of the road? Journal of Experimental Psychology: Human Perception and Performance, 38(3), 735-745.

Robertshaw, K. D., \& Wilkie, R. M. (2008). Does gaze influence steering around a bend? Journal of Vision, 8(4)(18), 1-13.

Ryan, T. P. (2009). Modern Regression Methods (Second Edi). Hoboken, New Jersey: John Wiley \& Sons, Inc.

Saleh, L., Chevrel, P., Mars, F., Lafay, J. F., \& Claveau, F. (2011). Human-like cybernetic driver model for lane keeping. Proceedings of the 18th World Congress of the International Federation of Automatic Control, 4368-4373.

Salvucci, D. D., \& Gray, R. (2004). A two-point visual control model of steering. Perception, 33(10), 1233-48.

Saunders, J., \& Ma, K. (2011). Can observers judge future circular path relative to a target from retinal flow? Journal of Vision, 11(7):16, 1-17.

Senders, J. W., Kristofferson, A. B., Levison, W. H., Dietrich, C. W., \& Ward, J. L. (1967). The attentional demand of automobile driving. Highway Research Record, 195, 15-33 
Sentouh, C., Chevrel, P., Mars, F., \& Claveau, F. (2009). A Sensoriomotor Driver Model for Steering Control. Proceedings of the 2009 IEEE International Conference on Systems, Man and Cybernetics (SMC), (October), 2462-2467.

Steen, J., Damveld, H. J., Happee, R., van Paassen, M. M., \& Mulder, M. (2011). A review of visual driver models for system identification purposes. 2011 IEEE International Conference on Systems, Man, and Cybernetics (SMC), 2093-2100.

Underwood, G., Chapman, P., Crundall, D. E., Cooper, S., \& Wallen, R. (1999). The visual control of steering and driving: where do we look when negotiating curves? In A. G. Gale, I. D. Brown, C. M. Haslegrave, \& S. P. Taylor (Eds.), Vision in Vehicles VII (pp. 245-252). Amsterdam: Elsevier.

van Leeuwen, P. M., Happee, R., \& de Winter, J. C. F. (2014). Vertical field of view restriction in driver training: A simulator-based evaluation. Transportation Research Part F, 24,

Vansteenkiste, P., Cardon, G., D'Hondt, E., Philippaerts, R., \& Lenoir, M. (2013). The visual control of bicycle steering: The effects of speed and path width. Accident Analysis and Prevention, 51, 222-7.

Wann, J. P., \& Swapp, D. K. (2000). Why you should look where you are going. Nature Neuroscience, 3(7), 647-8.

Warren, W. H., Kay, B. a, Zosh, W. D., Duchon, a P., \& Sahuc, S. (2001). Optic flow is used to control human walking. Nature Neuroscience, 4(2), 213-6.

Warren, W. H., Mestre, D. R., Blackwell, a W., \& Morris, M. W. (1991). Perception of circular heading from optical flow. Journal of Experimental Psychology. Human Perception and Performance, 17(1), 28-43.

Wilkie, R. M., Kountouriotis, G. K., Merat, N., \& Wann, J. P. (2010). Using vision to control locomotion: looking where you want to go. Experimental Brain Research, 204(4), 53947.

Wilkie, R. M., \& Wann, J. P. (2002). Driving as night falls: the contribution of retinal flow and visual direction to the control of steering. Current Biology, 12(23), 2014-7.

Wilkie, R. M., \& Wann, J. P. (2003a). Controlling steering and judging heading: Retinal flow, visual direction, and extraretinal information. Journal of Experimental Psychology: Human Perception and Performance, 29(2), 363-378.

Wilkie, R. M., \& Wann, J. P. (2003b). Eye-movements aid the control of locomotion. Journal of Vision, 3, 677-684.

Wilkie, R. M., \& Wann, J. P. (2005). The role of visual and nonvisual information in the control of locomotion. Journal of Experimental Psychology. Human Perception and Performance, 31(5), 901-11.

Wilkie, R. M., \& Wann, J. P. (2006). Judgments of path, not heading, guide locomotion. Journal of Experimental Psychology. Human Perception and Performance, 32(1), 88-96.

Wilkie, R. M., Wann, J. P., \& Allison, R. S. (2008). Active gaze, visual look-ahead, and locomotor control. Journal of Experimental Psychology. Human Perception and Performance, 34(5), 1150-64. 


\section{Appendices}

\section{Appendix A}

\section{Fitting Slopes across Flow Levels: Assessing Linear Fit}

Instead of approximating the gradient of the slope $\left(\left(\mathrm{FS}_{\mathrm{F}}-\mathrm{SL}_{\mathrm{F}}\right) / 2\right.$; as per Experiment 1$)$, FISB scores were calculated by finding the slope of the linear regression fitted to the steering bias estimates across the five flow speeds for each road condition. In Experiment 2, it quickly emerged that this linear relationship did not fit well across five flow levels. In particular, the shift in steering bias from $F L_{.5}$ to $F L_{.75}$ was clearly greater than the shift from $\mathrm{FL}_{1.25}$ to $\mathrm{FL}_{1.5}$, meaning that a simple linear fit did not capture the data well (Figure 13), with $R^{2}$ values ranging from around $4 \mathrm{in} \mathrm{N}_{\mathrm{Rd}}$, to around .75 in $\mathrm{NMF}_{\mathrm{Rd}}$. The next section investigates different approaches to obtaining an acceptable fit across flow levels.

\section{Fitting Slopes across Flow Levels: Logarithmic Transform}

It has been argued that Flow Speed perception follows a Weber relationship (Authié \& Mestre, 2012). Weber's law stipulates that perceived differences between two signals of difference magnitudes is determined by the ratio between the two signals, rather than the absolute difference. Therefore, given the same separation interval, two values of higher magnitude will have a smaller perceived difference than two values of smaller magnitudes.

In the current experiment, the ratio for $\mathrm{FL} .75$ and $F L_{.5}$ is 1.5 , but the ratio for $\mathrm{FL}_{1.5}$ and $\mathrm{FL}_{1.25}$ is 1.2. The larger ratio between lower flow speeds might explain why a greater shift in steering behaviour is observed than for higher levels. With this in mind, the flow levels were 
Running Head: Optic Flow modulates Guidance Steering Control

logarithmically transformed, so that $.5, .75,1,1.25$, and 1.5 became $-.301,-.125,0, .097$, and .176. A major advantage of doing a logarithmic transform on the flow values is that they can still be used to carry out linear regression. Whilst this led to minor improvements in $\mathrm{R}^{2}$ (Figure $13)$, the $R^{2}$ values remained low, indicating that this may still be a sub-optimal approach to capturing the true extent of FISB.

\section{Fitting Slopes across Flow Levels: Weighted Linear Regression}

An assumption of linear regression is that every data point contributes equally to the regression line (i.e. it assumes that every data point is equally reliable). In the present case, linear regression is conducted on 5 data points (the flow levels), each representing an average of 6 trials per person. If a participant was particularly variable in one condition, this would be weighted equally with a condition where the participant's behaviour was highly precise, even though the precise condition's estimate should really be trusted more. A Weighted Least Squares procedure takes into account the uncertainty in participant bias estimates (Ryan, 2009), and can be expressed mathematically as follows:

$$
\beta 1=\frac{\sum w_{i} x_{i} y_{i}-\frac{\left(\sum w_{i} x_{i}\right)\left(\sum w_{i} y_{i}\right)}{\sum w_{i}}}{\sum w_{i} x_{i}^{2}-\frac{\left(\sum w_{i} x_{i}\right)^{2}}{\sum w_{i}}}
$$

Using a weighted least squares regression approach improved the fit of FISB for every condition (Figure 13). However, an additional minor improvement was obtained from logarithmically transforming the scale (Figure 13). Therefore, a weighted least squares regression on a logarithmically transformed flow scale was the method chosen to calculate FISB scores for Experiment 2. Since a large slope value indicates that participants were biased 
by flow in the predicted direction (oversteering for faster-than-veridical, understeering for slower-than-veridical), the $\beta 1$ estimate was taken as the FISB score.

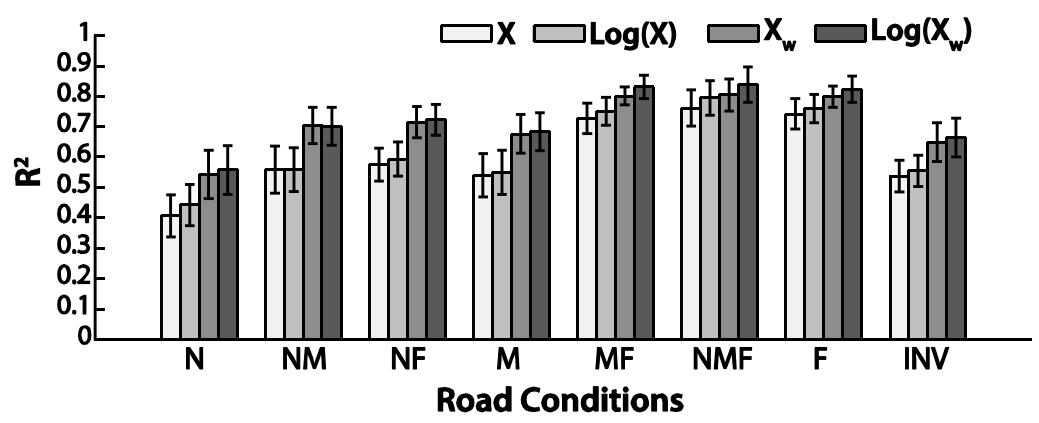

Figure 13. $\mathbf{R}^{2}$ estimates are calculated per person, per condition, and then subsequently averaged. The graph compares average explained variance for a 1) linear fit, a 2) linear fit on logarithmically transformed data, a 3) weighted linear fit, and a 4) weighted linear fit on logarithmically transformed data. Error bars represent standard error of the mean.

Despite the improvements to how FISB is calculated, it is important to note that it clearly does not fully capture the spread of flow levels, and the explanatory power of FISB varies between road conditions with $\mathrm{R}^{2}$ values ranging between .56 for $\mathrm{N}_{\mathrm{Rd}}$ and .84 for $\mathrm{NMF}_{\mathrm{Rd}}$ (Figure 13). This variation is in part due to a drop in $\mathrm{R}^{2}$ in conditions where the change in steering response to flow manipulation is small ( $\mathrm{N}_{\mathrm{Rd}}$; see Figure 13 ), therefore fitting a line is problematic, and a rise in $\mathrm{R}^{2}$ in conditions where flow manipulations cause distinct and consistent alterations to steering response $\left(\mathrm{NMF}_{\mathrm{Rd}}, \mathrm{F}_{\mathrm{Rd}}\right.$, and $\mathrm{MF}_{\mathrm{Rd}}$; see Figure 13$)$. Although this metric may be somewhat imprecise, it remains useful for facilitating comparisons across road conditions. 